\title{
Ultrafast radiationless transition pathways through conical intersections in photo-excited $9 \mathrm{H}$-adenine
}

\author{
Walid Mohamed Ibrahim Hassan, ${ }^{a}$ Wilfredo Credo Chung, $\dagger^{a}$ Noriyuki Shimakura, ${ }^{b}$ \\ Shiro Koseki, ${ }^{c}$ Hirohiko Kono ${ }^{a}$ and Yuichi Fujimura*ad
}

Received 10th December 2009, Accepted 11th February 2010

First published as an Advance Article on the web 31st March 2010

DOI: $10.1039 / \mathrm{b} 926102 \mathrm{~h}$

We performed CASSCF and MRCI calculations for determination of the effective pathways of ultrafast radiationless transitions from the optically allowed $\pi \pi^{*}{ }^{1} \mathrm{~L}_{\mathrm{a}}$ state to the ground state $\mathrm{S}_{0}$ of $9 \mathrm{H}$-adenine. The $n \pi^{*}, \pi \sigma^{*}$, and two $\pi \pi^{*}$ states were taken into account as states involved in the radiationless process. Optimized geometry and conical intersections were searched in the full dimensional space for the vibrational degrees of freedom by using the suite of quantum chemistry codes MOLPRO. The MRCI transition energies to excited states are in good agreement with the experimental values. The mechanisms of three competing pathways, two indirect pathways via the $\pi \sigma^{*}$ and $n \pi^{*}$ states, ${ }^{1} \mathrm{~L}_{\mathrm{a}} \rightarrow \pi \sigma^{*} \rightarrow \mathrm{S}_{0}$ and ${ }^{1} \mathrm{~L}_{\mathrm{a}} \rightarrow n \pi^{*} \rightarrow \mathrm{S}_{0}$, and a direct pathway ${ }^{1} \mathrm{~L}_{\mathrm{a}} \rightarrow \mathrm{S}_{0}$, were examined on the basis of the structures and energies of conical intersections involved in ultrafast radiationless transitions from ${ }^{1} \mathrm{~L}_{\mathrm{a}}$ to $\mathrm{S}_{0}$. Any conical intersection between the $\pi \sigma^{*}$ and $n \pi *$ states was not found. This suggests that the two indirect pathways are independent of each other. The $\pi \pi^{*}{ }^{1} \mathrm{~L}_{\mathrm{a}}-\pi \sigma^{*}$ conical intersection lies higher than the $\pi \pi^{*}{ }^{1} \mathrm{~L}_{\mathrm{a}}$ state at the Franck-Condon geometry by $0.19 \mathrm{eV}$ according to the present MRCI calculation, which is consistent with the experimental observation that a new channel is open at the excess energy of $\sim 0.2 \mathrm{eV}$ above the band origin of the $\pi \pi^{*}{ }^{1} \mathrm{~L}_{\mathrm{a}}$ state. It is concluded that relaxation from the $\pi \pi^{*}{ }^{1} \mathrm{~L}_{\mathrm{a}}-\pi \sigma^{*}$ conical intersection to $\mathrm{S}_{0}$ occurs mainly through the $\pi \sigma^{*}-\mathrm{S}_{0}$ conical intersection. The $\pi \pi^{*}{ }^{1} \mathrm{~L}_{\mathrm{a}}-n \pi^{*}$ conical intersection lies higher by $0.1 \mathrm{eV}$ (MRCI value) than the $\pi \pi^{*}{ }^{1} \mathrm{~L}_{\mathrm{a}}$ state at the Franck-Condon geometry. The fast decay component in time-resolved spectra of $9 \mathrm{H}$-adenine is attributed to rapid radiationless transitions to the $n \pi *$ state via this conical intersection followed by the transition to $\mathrm{S}_{0}$ via the $n \pi^{*}-\mathrm{S}_{0}$ ( or $\pi \pi^{*}{ }^{1} \mathrm{~L}_{\mathrm{a}}-\mathrm{S}_{0}$ ) conical intersection. The $\pi \pi^{*}{ }^{1} \mathrm{~L}_{\mathrm{a}}-\mathrm{S}_{0}$ conical intersection of large out-of-plane distortion has the lowest energy among the conical intersections found in this study. We identified the transition state between the $\pi \pi^{*}{ }^{1} \mathrm{~L}_{\mathrm{a}}$ at the Franck-Condon geometry and the $\pi \pi^{*}{ }^{1} \mathrm{~L}_{\mathrm{a}}-\mathrm{S}_{0}$ conical intersection. The MRCI energy of the transition state on the ${ }^{1} \mathrm{~L}_{\mathrm{a}}$ potential surface is higher by $0.21 \mathrm{eV}$ than the vertical excitation energy. The possibility of strong coupling between the two close-lying states ${ }^{1} \mathrm{~L}_{\mathrm{a}}$ and $n \pi *$ indicates that, besides this direct pathway, radiationless transitions to $\mathrm{S}_{0}$ via the $\pi \pi^{*}{ }^{1} \mathrm{~L}_{\mathrm{a}}-\mathrm{S}_{0}$ conical intersection can also occur after rapid relaxations between ${ }^{1} \mathrm{~L}_{\mathrm{a}}$ and $n \pi^{*}$. The analysis of the $\boldsymbol{h}$-vector for each conical intersection has shown that the active coupling for the $\pi \sigma^{*}$ pathway is dominated by the out-of-plane normal mode $\nu_{10}$, while the active coupling for the $n \pi^{*}$ pathway is distributed among many normal modes. Control of the branching ratio of the two indirect pathways can be achieved by selective excitation of single vibronic levels involving active coupling modes such as the mode $\nu_{10}$.

\section{Introduction}

Deoxyribo nucleic acid (DNA) bases are remarkably stable with respect to photochemical degradation. Although the

\footnotetext{
${ }^{a}$ Department of Chemistry, Graduate School of Science,

Tohoku University, Sendai, 980-8578, Japan.

E-mail: fujimurayuichi@mail.tains.tohoku.ac.jp

${ }^{b}$ Department of Chemistry, Niigata University,

Ikarashi Nino-cho 8050, Niigata, 950-2181, Japan

${ }^{c}$ Department of Chemistry, Graduate School of Science,

Osaka Prefecture University, Sakai, Osaka, 599-8531, Japan

${ }^{d}$ Department of Applied Chemistry, Institute of Molecular Science, National Chiao-Tung University, Hsin-Chu, 300, Taiwan,

Republic of China

$\dagger$ Present address: Fukui Institute for Fundamental Chemistry, Kyoto

University, 34-4 Takano-nishihirakicho, Kyoto 606-8103, Japan
}

DNA and RNA bases show a strong absorption in the range of 200-300 nm, these nucleobases are characterized by very small fluorescence quantum yields and remarkable stability against UV irradiation. ${ }^{1}$ This photostability is due to the existence of ultrafast radiationless transition pathways. ${ }^{2}$ After UV excitation, the bases decay within a subpicosecond time scale to vibrationally hot levels of the electronic ground state $\mathrm{S}_{0}$.

There have been numerous experimental and theoretical investigations on the mechanism of rapid decay of the photoexcited purine base adenine. ${ }^{2}$ The direct evidence of ultrafast decay of isolated $9 \mathrm{H}$-adenine has been shown by using mass-selected, femtosecond pump-probe spectroscopic methods such as resonantly enhanced multiphoton ionization (REMPI) spectroscopy. ${ }^{3}$ Optically created electronic energy 
transfers to vibrational and/or rotational energies in $\mathrm{S}_{0}$ through rapid internal conversion. This generally requires crossing of potential energy surfaces. In particular, conical intersections ${ }^{4}$ are known to greatly facilitate the internal conversion process. ${ }^{5,6}$

Sobolewski and Domcke, and their group proposed that internal conversion of $9 \mathrm{H}$-adenine takes place from the optically allowed $\pi \pi^{*}{ }^{1} \mathrm{~L}_{\mathrm{a}}$ state to the excited singlet state of $\pi \sigma^{*}$ character, which has a repulsive potential surface with respect to the $\mathrm{NH}$ bond stretching via a conical intersection, and then internal conversion proceeds from the repulsive $\pi \sigma^{*}$ to $\mathrm{S}_{0}$ via another conical intersection. ${ }^{7}$ This decay mechanism was supported by the results of nanosecond experiments on the observation of population of the $\pi \sigma^{*}$ state by detection of $\mathrm{H}$ atom fragments, which results from the dissociation of $9 \mathrm{H}$-adenine along the repulsive $\mathrm{NH}$ bond potential. ${ }^{8}$ To investigate the dynamics of $\mathrm{H}$-loss via the $\pi \sigma^{*}$ state, Wells, Roberts and Stavros irradiated adenine with nanosecond or femtosecond UV (266 nm) pulses and detected $\mathrm{H}^{+}$produced through multiphoton ionization of generated $\mathrm{H}$ fragments. ${ }^{9}$ They have suggested the existence of two channels for the generation of $\mathrm{H}$-fragments, namely, a femtosecond $\mathrm{H}$-production from the dissociative $\pi \sigma^{*}$ state as well as a nanosecond $\mathrm{H}$-production from the hot ground state after internal conversion.

The role of the optically forbidden $n \pi *$ state lying below $\pi \pi^{*}$ ${ }^{1} \mathrm{~L}_{\mathrm{a}}$ has now come to attention in addition to the $\pi \sigma^{*}$ state. Kan, Jung and Kim have concluded from the results of femtosecond pump-probe transient ionization time-of-flight mass spectroscopy that the $n \pi^{*}$ state, rather than the $\pi \sigma^{*}$ state, plays the role of a doorway state for internal conversion via a conical intersection to $\mathrm{S}_{0} \cdot{ }^{3 d}$ Ullrich et al. have observed strong coupling between the $\pi \pi^{* 1} \mathrm{~L}_{\mathrm{a}}$ and $n \pi *$ states of isolated $9 \mathrm{H}$-adenine in a molecular beam by using time-resolved photoelectron spectroscopy. ${ }^{3 e, g}$ In time-resolved ionization signals for adenine in the gas phase, biexponential decays have been observed; the two components consist of a fast component with a lifetime of 40-100 fs and slow component with a lifetime of $0.75-1$ ps. ${ }^{10}$ The fast and slow components are assigned as the decays of the $\pi \pi^{*}{ }^{1} \mathrm{~L}_{\mathrm{a}}$ and $n \pi^{*}$ states, respectively.

A number of theoretical works have been devoted to investigate the roles of $n \pi^{*}$ and $\pi \sigma^{*}$ states. Perun, Sobolewski and Domcke have investigated the mechanisms responsible for the rapid deactivation of the $\pi \pi^{*}$ and $n \pi^{*}$ states by using the complete-active-space-self-consistent-field (CASSCF) and second-order perturbation theory based on the CASSCF reference (CASSCF/CASPT2). ${ }^{11}$ They have also presented semi-quantitative results on the mechanisms of the radiationless deactivation of the excited states $\pi \pi^{*}, n \pi^{*}$ and $\pi \sigma^{*} .^{11}$ Chen and $\mathrm{Li}$ have obtained the geometrical structures and potential energy surfaces associated with $\pi \pi^{*}$ and $n \pi^{*}$ states (including the optically forbidden $\pi \pi^{*}{ }^{1} \mathrm{~L}_{\mathrm{b}}$ state) by performing CASSCF/CASPT2 calculations with the 6-311G* basis set and active spaces up to $(14,11) .{ }^{12}$ Chin et al. ${ }^{13}$ have carried out CASSCF calculations to analyze the results of resonant two-photon ionization and resonance fluorescence spectra of $9 \mathrm{H}$-adenine in terms of the $\mathrm{S}_{0}, \pi \pi^{*}$, and $n \pi^{*}$ states. They have also calculated internal conversion rate constants from single- vibronic levels. Blancafort has searched minimum energy pathways via the $\pi \pi^{*}{ }^{1} \mathrm{~L}_{\mathrm{b}}$ and $n \pi *$ states from ${ }^{1} \mathrm{~L}_{\mathrm{a}}$ to $\mathrm{S}_{0}$ and calculated the energy barriers to conical intersections at the CASSCF/CASPT2 level. ${ }^{14} \mathrm{He}$ has reported the existence of the indirect path via the ${ }^{1} \mathrm{~L}_{\mathrm{b}}$ state coupled to the $n \pi *$ state.

Recently, rather different hypotheses have been raised to explain the origin of the biexponential decay with fast and slow components in time-resolved spectra in the gas phase. ${ }^{10}$ On the basis of calculations of reaction paths at CASPT2 level, Serrano-Andrés and co-workers ${ }^{15}$ proposed for $9 \mathrm{H}$-adenine that the fast component corresponds to a fast deactivation of a fraction of the population passing through the conical intersection connecting the ${ }^{1} \mathrm{~L}_{\mathrm{a}}$ and $\mathrm{S}_{0}$ states. They claimed that this pathway is barrierless. Fabiano and Thiel have performed dynamics calculations based on the surface-hopping method to describe the nonadiabatic dynamics after vertical excitation to the ${ }^{1} \mathrm{~L}_{\mathrm{a}}$ state within a semiempirical OM2/MR-CI level of theory. ${ }^{16}$ No equilibrium geometry could be found for the ${ }^{1} \mathrm{~L}_{\mathrm{a}}$ state, because energy minimizations starting from the equilibrium geometry of the ground state (the Franck-Condon geometry) led directly to the ${ }^{1} \mathrm{~L}_{\mathrm{a}}-\mathrm{S}_{0}$ conical intersection. According to their results, the minimal-energy path also crosses the $n \pi *$ state on the downhill toward the ${ }^{1} \mathrm{~L}_{\mathrm{a}}-\mathrm{S}_{0}$ conical intersection. Therefore, contrary to the direct mechanism proposed by Serrano-Andrés et al., most trajectories follwed the rapid deexcitation pathway (fast component of $15 \mathrm{fs}$ ) through the ${ }^{1} \mathrm{~L}_{\mathrm{a}}-n \pi *$ conical intersection, and proceeded to the $n \pi^{*}-\mathrm{S}_{0}$ conical intersection (slow component of $560 \mathrm{fs}$ ). The fast decay component is attributed to the direct decay through the ${ }^{1} \mathrm{~L}_{\mathrm{a}}-n \pi *$ conical intersection. Only a small number of trajectories transfer to $\mathrm{S}_{0}$ via the ${ }^{1} \mathrm{~L}_{\mathrm{a}}-\mathrm{S}_{0}$ conical intersection. Barbatti and Lischka ${ }^{17}$ have performed classical dynamics simulations on the potential surfaces obtained by a MR-CIS level of calculation. They also could not find clear evidence of direct deactivation from ${ }^{1} \mathrm{~L}_{\mathrm{a}}$ to $\mathrm{S}_{0}$.

On the other hand, on the basis of time-resolved photoelectron spectroscopy of $9 \mathrm{H}$-adenine, Satzger and co-workers ${ }^{10 a, 3 g}$ claim that for the $267 \mathrm{~nm}$ excitation the fast and slow components correspond to the population transfer to the $\pi \sigma^{*}$ state and the subsequent decay into the $S_{0}$ state through the NH stretching conical intersection, respectively. At lower wavelengths the importance of this path would decrease, given the availability of other channels. However, the results of dynamics simulation by Barbatti and Lischka ${ }^{17}$ indicated that there were no conical intersections associated with $\mathrm{NH}$ bond breaking. Thus, the radiationless transition mechanisms of photo-excited $9 \mathrm{H}$-adenine are not clarified, although significant theoretical attempts have been carried out.

In our previous study, ${ }^{18}$ we focused only on the role of the $\pi \sigma^{*}$ state in the ultrafast deactivation processes through conical intersections within a two-dimensional model. In this paper, we discuss also the role of the $n \pi *$ state on the basis of the results of CASSCF and MRCI calculations for the structures and energies related to ultrafast radiationless transitions through conical intersections. We set the active space so that all the relevant states, namely, the $n \pi^{*}, \pi \sigma^{*}$, and two $\pi \pi^{*}$ states are included and made a full dimensional geometry search for the vibrational degrees of freedom of 9H-adenine. 
From the search of conical intersections, we show in this paper that three competing pathways are involved in ultrafast radiationless transitions from ${ }^{1} \mathrm{~L}_{\mathrm{a}}$ to $\mathrm{S}_{0}$; two indirect pathways via the $\pi \sigma^{*}$ and $n \pi *$ states, ${ }^{1} \mathrm{~L}_{\mathrm{a}} \rightarrow \pi \sigma^{*} \rightarrow \mathrm{S}_{0}$ and ${ }^{1} \mathrm{~L}_{\mathrm{a}} \rightarrow n \pi^{*} \rightarrow \mathrm{S}_{0}$, and a direct pathway ${ }^{1} \mathrm{~L}_{\mathrm{a}} \rightarrow \mathrm{S}_{0}$. In the next section, our theoretical and computational treatments are briefly described. The tuning and coupling modes, denoted by the $\boldsymbol{g}$ - and $\boldsymbol{h}$-vectors, respectively, characterize the conical intersections of polyatomic molecules. The $\boldsymbol{g}$ - and $\boldsymbol{h}$-vectors defined for a conical intersection between two electronic states are outlined. ${ }^{19}$ Conical intersections involving three electronic states were not considered, though the optimization of conical intersections is expected to be improved. ${ }^{20}$ In section 3 , first, the results of quantum chemical calculations for the optimized structures and optical transition energies are presented. Then, the results of searching of conical intersections obtained by using MOLPRO $^{21}$ are described. Three fundamental radiationless pathways from the optically excited $\pi \pi^{*}{ }^{1} \mathrm{~L}_{\mathrm{a}}$ state to $\mathrm{S}_{0}$ are theoretically confirmed: the direct pathway and two indirect pathways via doorway $\pi \sigma^{*}$ and $n \pi *$ states. To prove that the conical intersections obtained are true ones, we have evaluated the geometric (Berry) phase effects ${ }^{22}$ on the adiabatic electronic wavefunctions. Finally, a control scheme of radiationless transition pathways from the ${ }^{1} \mathrm{~L}_{\mathrm{a}}$ state is proposed on the basis of the analysis of the $\boldsymbol{h}$-vectors (coupling modes) of conical intersections.

\section{Theoretical and computational treatments}

\subsection{Brief description of conical intersection}

Let us consider a case in which two electronic states ( $a$ and $b$ ) intersect at a position $\boldsymbol{R}_{c} \cdot{ }^{20}$ We denote the conical intersection between the $a$ and $b$ states by the $a-b$ conical intersection hereafter. Adiabatic electronic wavefunctions $\Psi_{a}(\boldsymbol{r}, \boldsymbol{R})$ and $\Psi_{b}(\boldsymbol{r}, \boldsymbol{R})$, in which $\boldsymbol{r}$ and $\boldsymbol{R}$ represent electronic and nuclear coordinates respectively, are expanded in terms of diabatic electronic wave functions $\psi_{\alpha}(\boldsymbol{r}, \boldsymbol{R})$ and $\psi_{\beta}(\boldsymbol{r}, \boldsymbol{R})$ as

$$
\left(\begin{array}{c}
\Psi_{a}(\boldsymbol{r}, \boldsymbol{R}) \\
\Psi_{b}(\boldsymbol{r}, \boldsymbol{R})
\end{array}\right)=\left(\begin{array}{cc}
\cos \frac{\Theta(R)}{2} & -\sin \frac{\Theta(\boldsymbol{R})}{2} \\
\sin \frac{\Theta(R)}{2} & \cos \frac{\Theta(\boldsymbol{R})}{2}
\end{array}\right)\left(\begin{array}{l}
\psi_{\alpha}(\boldsymbol{r}, \boldsymbol{R}) \\
\psi_{\beta}(\boldsymbol{r}, \boldsymbol{R})
\end{array}\right)
$$

where $\Theta(\boldsymbol{R})$ is expressed in terms of the matrix elements, $H_{\alpha \alpha}(\boldsymbol{R}), H_{\beta \beta}(\boldsymbol{R})$ and $H_{\alpha \beta}(\boldsymbol{R})\left(=\left\langle\psi_{\alpha}(\boldsymbol{r}, \boldsymbol{R})|H(\boldsymbol{r}, \boldsymbol{R})| \psi_{\beta}(\boldsymbol{r}, \boldsymbol{R})\right\rangle_{\boldsymbol{r}}\right)$ of the electronic Hamiltonian at $\boldsymbol{R}, H(\boldsymbol{r}, \boldsymbol{R})$ :

$$
\tan \Theta(\boldsymbol{R})=\frac{2 H_{\alpha \beta}(\boldsymbol{R})}{H_{\alpha \alpha}(\boldsymbol{R})-H_{\beta \beta}(\boldsymbol{R})} .
$$

The electronic Hamiltonian is given by $H(\boldsymbol{r}, \boldsymbol{R})=T_{r}+U(\boldsymbol{r}, \boldsymbol{R})$, where $T_{r}$ is the kinetic energy of electrons and $U(\boldsymbol{r}, \boldsymbol{R})$ represents the electrostatic interactions among electrons and nuclei.

The adiabatic potential energy surfaces near the intersection point can be obtained by solving

$$
\left|\begin{array}{cc}
H_{\alpha \alpha}(\boldsymbol{R})-E(\boldsymbol{R}) & H_{\alpha \beta}(\boldsymbol{R}) \\
H_{\beta \alpha}(\boldsymbol{R}) & H_{\beta \beta}(\boldsymbol{R})-E(\boldsymbol{R})
\end{array}\right|=0,
$$

as

$$
\begin{aligned}
E_{a \text { or } b}(\boldsymbol{R})= & \frac{1}{2}\left\{H_{\alpha \alpha}(\boldsymbol{R})+H_{\beta \beta}(\boldsymbol{R})\right. \\
& \left. \pm \sqrt{\left[H_{\alpha \alpha}(\boldsymbol{R})-H_{\beta \beta}(\boldsymbol{R})\right]^{2}+4\left|H_{\alpha \beta}(\boldsymbol{R})\right|^{2}}\right\}
\end{aligned}
$$

The $a-b$ conical intersection is determined by the condition that the energy difference between the two adiabatic electronic states is equal to zero, that is,

$$
\left[H_{\alpha \alpha}(\boldsymbol{R})-H_{\beta \beta}(\boldsymbol{R})\right]^{2}+4\left|H_{\alpha \beta}(\boldsymbol{R})\right|^{2}=0 .
$$

The difference between the two diagonal matrix elements can be expanded to the first order of internal coordinates $\{\boldsymbol{Q}\}$ around a position $\boldsymbol{R}=\boldsymbol{R}^{*}$ that locates close to the intersection position $\boldsymbol{R}=\boldsymbol{R}_{\mathrm{c}}$ as

$$
\begin{aligned}
& H_{\alpha \alpha}(\boldsymbol{R})-H_{\beta \beta}(\boldsymbol{R}) \\
& \approx H_{\alpha \alpha}\left(\boldsymbol{R}^{*}\right)-H_{\beta \beta}\left(\boldsymbol{R}^{*}\right)+\sum_{i} \frac{\partial}{\partial Q}\left[H_{\alpha \alpha}(\boldsymbol{R})-H_{\beta \beta}(\boldsymbol{R})\right]_{\boldsymbol{R}=\boldsymbol{R}^{*}} Q_{i} \\
& =H_{\alpha \alpha}\left(\boldsymbol{R}^{*}\right)-H_{\beta \beta}\left(\boldsymbol{R}^{*}\right)+\boldsymbol{g} \cdot \boldsymbol{Q} .
\end{aligned}
$$

Here, the $g$-vector represents the difference in the gradient of the diabatic potential energy between the two electronic states at position $\boldsymbol{R}=\boldsymbol{R}^{*}$ :

$$
\begin{aligned}
\boldsymbol{g} \equiv & {\left[\left\langle\psi_{\alpha}(\boldsymbol{r}, \boldsymbol{R})|\nabla H(\boldsymbol{r}, \boldsymbol{R})| \psi_{\alpha}(\boldsymbol{r}, \boldsymbol{R})\right\rangle\right.} \\
& \left.-\left\langle\psi_{\beta}(\boldsymbol{r}, \boldsymbol{R})|\nabla H(\boldsymbol{r}, \boldsymbol{R})| \psi_{\beta}(\boldsymbol{r}, \boldsymbol{R})\right\rangle\right]_{\boldsymbol{R}}=\boldsymbol{R}^{*} .
\end{aligned}
$$

The internal coordinates $\{\boldsymbol{Q}\}$ involved in the $\boldsymbol{g}$-vector, $\left\{\boldsymbol{Q}_{t}\right\}$, are referred to as tuning or accepting modes in the following discussion. The off-diagonal matrix element is expanded in the similar way described above as

$$
\begin{aligned}
H_{\alpha \beta}(\boldsymbol{R}) & \approx H_{\alpha \beta}\left(\boldsymbol{R}^{*}\right)+\left.\sum_{i} \frac{\partial}{\partial Q_{i}} H_{\alpha \beta}(\boldsymbol{R})\right|_{\boldsymbol{R}=\boldsymbol{R}^{*}} Q_{i} \\
& =H_{\alpha \beta}\left(\boldsymbol{R}^{*}\right)+\boldsymbol{h} \cdot \boldsymbol{Q} .
\end{aligned}
$$

Here, the $\boldsymbol{h}$-vector is defined as

$$
\boldsymbol{h} \equiv\left\langle\psi_{\alpha}(\boldsymbol{r}, \boldsymbol{R})|\nabla H(\boldsymbol{r}, \boldsymbol{R})| \psi_{\beta}(\boldsymbol{r}, \boldsymbol{R})\right\rangle_{\boldsymbol{R}}=\boldsymbol{R}^{*} .
$$

The internal coordinates involved in the $\boldsymbol{h}$-vector, $\left\{\boldsymbol{Q}_{\mathrm{c}}\right\}$, are called coupling or promoting modes.

When we find the conical intersection $\boldsymbol{R}^{*}=\boldsymbol{R}_{\boldsymbol{c}}$, eqn (6) and (8) with eqn (7) and (9) are evaluated at $\boldsymbol{R}=\boldsymbol{R}_{\mathrm{c}}$ as

$$
H_{\alpha \alpha}(\boldsymbol{R})-H_{\beta \beta}(\boldsymbol{R}) \approx \boldsymbol{g} \cdot \boldsymbol{Q}
$$

and

$$
H_{\alpha \beta}(\boldsymbol{R}) \approx \boldsymbol{h} \cdot \boldsymbol{Q}
$$

We now define $d$ as the energy difference between the two electronic states, $a$ and $b$, near $\boldsymbol{R}_{\mathrm{c}}$. Then, from eqn (4) we obtain

$$
\left[H_{\alpha \alpha}(\boldsymbol{R})-H_{\beta \beta}(\boldsymbol{R})\right]^{2}+4\left|H_{\alpha \beta}(\boldsymbol{R})\right|^{2}=d^{2} .
$$


Defining $d_{t}=\boldsymbol{g} \cdot \boldsymbol{Q}$ and $d_{c}=\boldsymbol{h} \cdot \boldsymbol{Q}$, and using eqn (10) and (11), we rewrite eqn (12) as

$$
d_{t}^{2}+\left(2 d_{c}\right)^{2}=d^{2}
$$

and

$$
\tan \theta=\frac{2 d_{c}}{d_{t}}
$$

Eqn (13) and (14) express a circle for $\{\boldsymbol{Q}\}$ with radius $d$ and argument (angle) $\theta$ if these tuning coordinates $\left\{\boldsymbol{Q}_{t}\right\}$ and coupling coordinates $\left\{\boldsymbol{Q}_{\mathrm{c}}\right\}$ are perpendicular to each other. From a symmetry consideration this condition is satisfied when $9 \mathrm{H}$-adenine is planar at the $a-b$ conical intersection. That is, for a conical intersection at a planar geometrical structure, the $\boldsymbol{g}$-vector and $\boldsymbol{h}$-vector are perpendicular to each other because the $g$-vector belongs to $\mathrm{A}^{\prime}$ of the point group Cs, while the $\boldsymbol{h}$-vector belongs to $\mathrm{A}^{\prime \prime}$. Therefore, $\boldsymbol{g}$ - and $\boldsymbol{h}$-vectors can be used as orthogonal bases for rotation as shown in eqn (13) and (14). The Berry phase effects are another proof of conical intersection: the adiabatic electronic wavefunctions associated with the conical intersection change its sign (geometric Berry phase) when they are rotated along a circle around the conical intersection.

Generally speaking, for a conical intersection possessing no symmetry, the $\boldsymbol{g}$ - and $\boldsymbol{h}$-vectors are not perpendicular to each other. For example, the $\pi \pi^{*}{ }^{1} \mathrm{~L}_{\mathrm{a}}$ and $\mathrm{S}_{0}$ have the same symmetry of the Cs point group and their potential energy curves are usually avoided to run across each other. However, it is known that a conical intersection is found in the coordinate space of nonsymmetric structures (see section 3.6) and two orthgonalized vectors should be constructed for the evaluation of Berry phase effects. When two electronic states are degenerate at a conical intersection, any linear combination of the electronic wavefunctions satisfies the electronic Schrödinger equation. Yarkony ${ }^{19 b, c}$ proposed that a set of orthogonal tuning and coupling vectors can be provided by diagonalization of the following two-dimensional matrix at a conical intersection $\boldsymbol{R}_{\boldsymbol{c}}$ :

$$
\left(\begin{array}{ll}
\langle\alpha|\nabla H(\boldsymbol{r}, \boldsymbol{R})| \alpha\rangle & \langle\alpha|\nabla H(\boldsymbol{r}, \boldsymbol{R})| \beta\rangle \\
\langle\beta|\nabla H(\boldsymbol{r}, \boldsymbol{R})| \alpha\rangle & \langle\beta|\nabla H(\boldsymbol{r}, \boldsymbol{R})| \beta\rangle
\end{array}\right)_{\boldsymbol{R}=\boldsymbol{R}_{c}},
$$

where the electronic wave functions $|\alpha\rangle$ and $|\beta\rangle$ are given by $\left|\psi_{\alpha}(\boldsymbol{r}, \boldsymbol{R})\right\rangle$ and $\left|\psi_{\beta}(\boldsymbol{r}, \boldsymbol{R})\right\rangle$, respectively.

\subsection{Computational details}

2.2.1 Electronic structure calculations. In this study, we employed the $\mathrm{CASSCF}^{23}$ level of theory with the $6-31++\mathrm{G}^{* *}$ basis set to calculate the energies of electronic states and to optimize the geometrical structures of the conical intersections between two states. The CASSCF active space for $9 \mathrm{H}$-adenine includes six $\pi$, one $\sigma$, and one lone pair orbitals and eight electrons. Three $\pi$ and one lone pair orbitals are occupied in the ground-state configuration. The active orbitals at the equilibrium structure of $\mathrm{S}_{0}$ are illustrated in Fig. 1. The state-average technique was employed; the electron density averaged over five electronic states, $\mathrm{S}_{0}, \pi \pi^{*}{ }^{1} \mathrm{~L}_{\mathrm{b}}, \pi \pi^{* 1} \mathrm{~L}_{\mathrm{a}}, \pi \sigma^{*}$, and $n \pi *$ is used in the orbital optimization. An internally contracted multi-reference configuration interaction (MRCI)

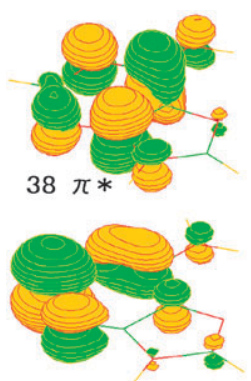

$36 \pi$

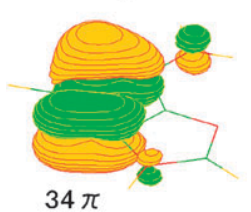

$34 \pi$
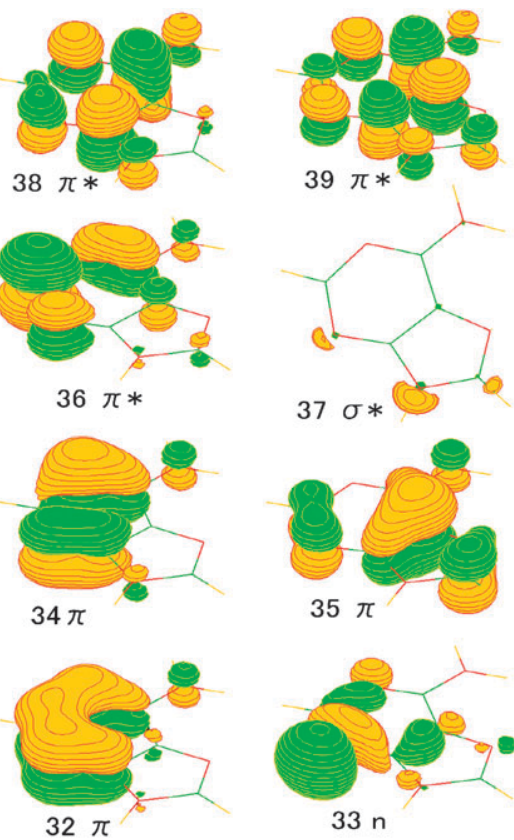

$37 \sigma$
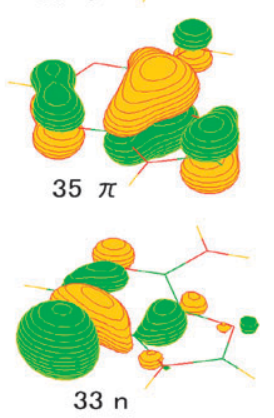

Fig. 1 Eight molecular orbitals defining the CASSCF active space at the equilibrium structure of $\mathrm{S}_{0}$. The contour plot of the density of the $\sigma^{*}$ orbital has been drawn at intervals of 0.035 e $\AA^{-3}$ instead of the standard value of $0.05 \mathrm{e}^{-3}$ using MOLDEN program. ${ }^{25}$

method $^{24}$ was used to improve the accuracy of the energies obtained by CASSCF.

2.2.2 Linearly interpolated internal coordinate (LIIC) reaction paths. Having optimized the positions of the local minima of the excited states and the conical intersections, we constructed linearly interpolated internal coordinate (LIIC) ${ }^{9 b, 26}$ reaction paths. The LIIC path in the multidimensional internalcoordinate space, which is equivalent to the linear synchronous transit path, is defined as the straight line which connects a given initial local minimum to a given conical intersection (or vice verse). Energy calculations have been performed at 100 points along each LIIC path to obtain the reaction-path potential-energy profiles and to evaluate the activation potential energy.

\section{Results and discussion}

\subsection{Optimized geometrical structures in the ground and excited states and excitation energies}

The geometrical structures in the ground and low-lying excited states were optimized by using the state average CASSCF calculation. The vertical and adiabatic excitation energies have been refined by a single-point $\mathrm{MRCI}^{24}$ calculation. No symmetry constraint was imposed. The optimized structure of the ground state is nonplanar. However, we found for the ground state that the pyramidization of the amino group of adenine only slightly reduces the total energy. It is recognized, actually, that this pyramidization has a very minor effect on the energies of ground and excited states (of the order of $\left.0.125-0.015 \mathrm{kcal} \mathrm{mol}^{-1}\right) .^{27}$

Optimized bond lengths and bond angles are shown in Fig. 2, which agree reasonably well with experimental ${ }^{28}$ and 


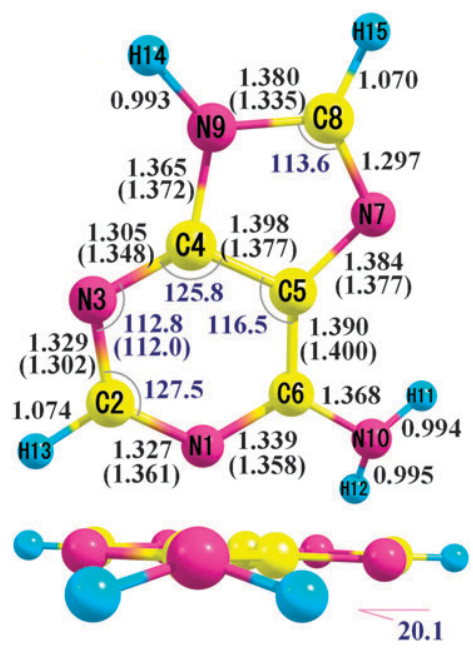

Fig. 2 Optimized $\mathrm{C}_{1}$ geometry of $9 \mathrm{H}$-adenine in the ground state $\mathrm{S}_{0}$. Bond lengths are given in angstroms and angles are in degrees. The values in parentheses are experimental ones. ${ }^{28 a}$

Table 1 Computed excitation energies in $\mathrm{eV}$ for $9 \mathrm{H}$-adenine at $\mathrm{C}_{1}$ symmetry. The values in parentheses are calculated by using MRCI and the values in the column "Exp." are experimental ones

\begin{tabular}{|c|c|c|c|c|c|c|}
\hline \multirow[b]{3}{*}{ State } & \multicolumn{3}{|c|}{ Vertical excitation/eV } & \multicolumn{3}{|c|}{ Adiabatic excitation/eV } \\
\hline & \multicolumn{2}{|l|}{ Theoretical } & \multirow{2}{*}{ Exp. } & \multicolumn{2}{|l|}{ Theoretical } & \multirow{2}{*}{ Exp. ${ }^{11 b, 15 b}$} \\
\hline & This work & Other & & This work & Other & \\
\hline${ }^{1} \mathrm{~L}_{\mathrm{b}}$ & $6.19(4.75)$ & $4.90^{12}$ & $4.6^{1 b, 15 b}$ & $5.32(4.72)$ & $4.83^{15}$ & 4.48 \\
\hline$n \pi^{*}$ & $7.03(4.89)$ & $5.01^{12}$ & & $5.29(4.59)$ & $4.52^{15}$ & 4.40 \\
\hline${ }^{1} \mathrm{~L}_{\mathrm{a}}$ & $7.10(5.13)$ & $5.17^{17}$ & $5.12^{17}$ & $5.72(5.02)$ & & \\
\hline$\pi \sigma^{*}$ & $8.20(5.92)$ & & & $5.76(5.10)$ & & \\
\hline
\end{tabular}

theoretical $^{29}$ results. The dihedral angle H12N10C6N1 (out-of-plane angle) is $20.1^{\circ}$, in good agreement with the previously calculated values $\left(14.8-21.7^{\circ}\right)^{27}$ with different basis sets at MP2 level. The computed dipole moment of the ground state is $2.5 \mathrm{D}$. This is in good agreement with the experimental results of crystalline 9-methyladenine ${ }^{30}(2.4 \mathrm{D})$ and 9-butyladenine in solution ${ }^{31}(3.0 \pm 0.2 \mathrm{D})$ as well as with the results of previous calculations. ${ }^{11 b}$

The vertical and adiabatic excitation energies are summarized in Table 1. It is well-known that the optically allowed $\pi \pi^{* 1} \mathrm{~L}_{\mathrm{a}}$ state lies higher in energy than the optically forbidden $\pi \pi^{* 1} \mathrm{~L}_{\mathrm{b}}$ state. The vertical excitation energy to the $\pi \pi^{*}{ }^{1} \mathrm{~L}_{\mathrm{a}}$ state, $5.13 \mathrm{eV}$, which is calculated at the MRCI level of theory, is acceptable compared to the experimental data 4.9-5.2 $\mathrm{eV}^{15,17,32}$ and pervious theoretical calculations. ${ }^{33}$

The adiabatic excitation energy for the ${ }^{1} \mathrm{~L}_{\mathrm{b}}$ state calculated at the MRCI level, $4.72 \mathrm{eV}$, is within the range of previously calculated values $4.674 \mathrm{eV}^{13}$ and $4.83 \mathrm{eV} .{ }^{15}$ The calculated adiabatic excitation energy for the $n \pi^{*}$ state, which is adiabatically the lowest excited state, is $4.59 \mathrm{eV}$. This value is close to previously reported theoretical values $4.476 \mathrm{eV}^{11 b}$ and $4.52 \mathrm{eV},{ }^{15}$ and it is in good agreement with the experimental value $4.4 \mathrm{eV} .^{3 d}$ The potential minimum of the $\pi \pi^{* 1} \mathrm{~L}_{\mathrm{a}}$ state, $5.02 \mathrm{eV}(247 \mathrm{~nm})$, is only slightly lower than the vertical excitation energy of $5.13 \mathrm{eV}$. We found a potential minimum in the $\pi \sigma^{*}$ state, of which the energy is much lower than the
Table 2 Energies and point groups of $9 \mathrm{H}$-adenine at the conical intersections involved in the radiationless transitions from the ${ }^{1} \mathrm{~L}_{\mathrm{a}}$ state to the ground state $\mathrm{S}_{0}$. The origin of the energy is the potential minimum of $\mathrm{S}_{0}$. The values are obtained by a CASSCF level of calculation and the values in parentheses are obtained by MRCI. The symbol in the square bracket denotes the point group of the $9 \mathrm{H}$-adenine at the conical intersection

\begin{tabular}{lll}
\hline $\begin{array}{l}\text { Indirect pathway } \\
\mathrm{A} \rightarrow \mathrm{B} \rightarrow \mathrm{C}\end{array}$ & $\begin{array}{l}\text { Energy/eV } \\
\text { Conical intersection } \\
(\mathrm{A} \leftrightarrow \mathrm{B})\end{array}$ & $\begin{array}{l}\text { Conical intersection } \\
(\mathrm{B} \leftrightarrow \mathrm{C})\end{array}$ \\
\hline${ }^{1} \mathrm{~L}_{\mathrm{a}} \rightarrow \pi \sigma^{*} \rightarrow \mathrm{S}_{0}$ & $5.80(5.32)[\mathrm{Cs}]$ & $4.69(4.13)[\mathrm{Cs}]$ \\
${ }^{1} \mathrm{~L}_{\mathrm{a}} \rightarrow n \pi^{*} \rightarrow \mathrm{S}_{0}$ & $5.82(5.23)[\mathrm{Cs}]$ & $4.11(3.74)\left[\mathrm{C}_{1}\right]$ \\
Direct pathway & Energy at the conical intersection/eV \\
\hline${ }^{1} \mathrm{~L}_{\mathrm{a}} \rightarrow \mathrm{S}_{0}$ & $3.83(3.50)\left[\mathrm{C}_{1}\right]$ & \\
\hline
\end{tabular}

energy at the Franck-Condon geometry (by $0.82 \mathrm{eV}$ in the MRCI calculation). For N9-H14 bond stretching, the potential energy increases from the minimum to a low barrier and becomes repulsive, as shown in previous papers. ${ }^{11,18}$ The barrier height is less than $0.2 \mathrm{eV}$.

\subsection{Radiationless transition pathways}

Conical intersections related to the radiationless transitions from the $\pi \pi^{*}{ }^{1} \mathrm{~L}_{\mathrm{a}}$ state to the ground state $\mathrm{S}_{0}$ were located by using MOLPRO. The results are summarized in Table 2.

Let us now consider a radiationless transition pathway involving two intermediate electronic states (diabatic $\pi \sigma^{*}$ and $n \pi^{*}$ states). Here, we note that the coupling between the two electronic states can safely be omitted since the corresponding $\boldsymbol{h}$-vector is zero or negligibly small. This is because all the molecular orbitals are different and their overlap integrals are very small. For $9 \mathrm{H}$-adenine of Cs point group, the $\boldsymbol{h}$-vector is zero from symmetry. In fact, we could not find any conical intersections between the $\pi \sigma^{*}$ and $n \pi^{*}$ states. Hence, in the following discussion, two pathways will be treated independently: (1) $\pi \sigma^{*}$ pathway: a radiationless transition process via the $\pi \sigma^{*}$ state, and (2) $n \pi^{*}$ pathway: a radiationless transition process via the $n \pi *$ state. We note in Table 2 that there is no significant difference in energy between the $\pi \pi^{*}$ ${ }^{1} \mathrm{~L}_{\mathrm{a}}-n \pi *$ and $\pi \pi^{*}{ }^{1} \mathrm{~L}_{\mathrm{a}}-\pi \sigma^{*}$ conical intersections. This suggests that both the pathways may make an important contribution to the ultrafast radiationless transition of $9 \mathrm{H}$-adenine.

Each pathway is conveniently divided into two stages: the activation stage and the transfer stage. The first stage (activation stage) involves the range from the Franck-Condon region to the first conical intersection, and the subsequent stage (transfer stage) involves the range from the first conical intersection to the second one.

\subsection{The $\pi \sigma^{*}$ pathway}

3.3.1 The activation stage. Let us consider the activation stage of the $\pi \sigma^{*}$ pathway, namely, the stage from the optically allowed $\pi \pi^{*}{ }^{1} \mathrm{~L}_{\mathrm{a}}$ state to the $\pi \pi^{* 1} \mathrm{~L}_{\mathrm{a}}-\pi \sigma^{*}$ conical intersection. Fig. 3a shows the geometrical structure at this conical intersection. The bond length $R_{\mathrm{N} 9 \mathrm{H}}$ between $\mathrm{N} 9$ and $\mathrm{H} 14$ is $0.993 \AA$, which is consistent with the CASSCF value $R_{\mathrm{N} 9 \mathrm{H}}=0.988 \AA$ in our previous two-dimensional model. ${ }^{18}$ In both our studies, the geometrical structure at the $\pi \pi^{*}$ 

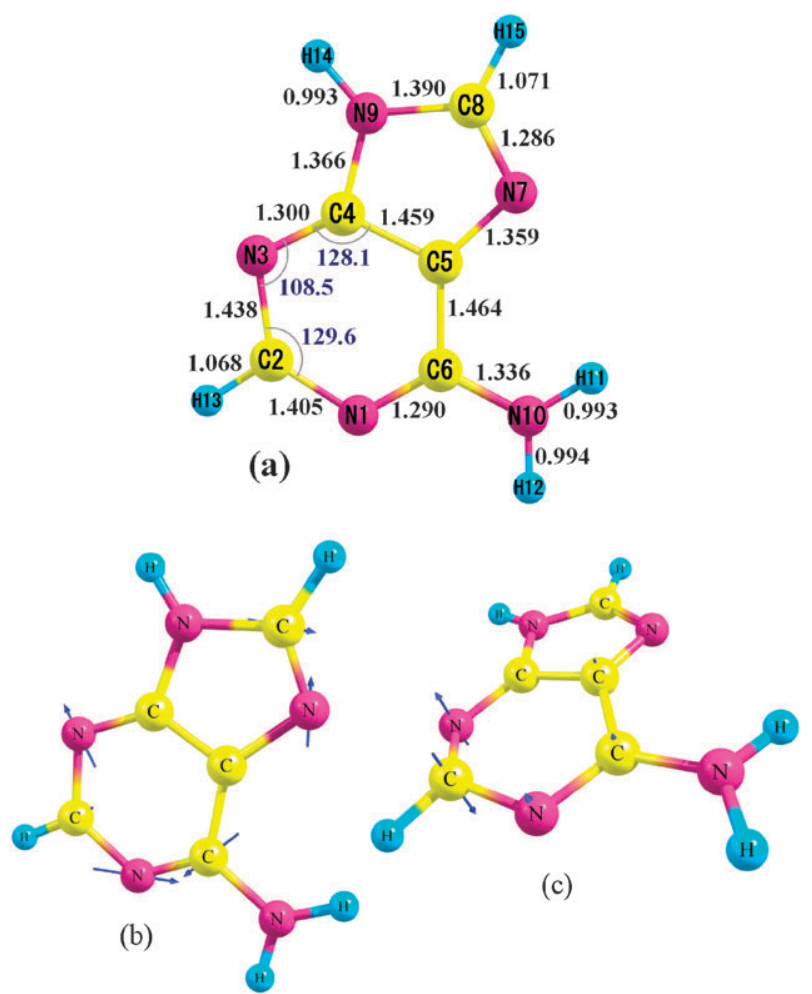

Fig. 3 (a) Cs structure at the $\pi \pi^{*}{ }^{1} \mathrm{~L}_{\mathrm{a}}-\pi \sigma^{*}$ conical intersection (b) the corresponding $\boldsymbol{g}$-vector and (c) $\boldsymbol{h}$-vector.

${ }^{1} \mathrm{~L}_{\mathrm{a}}-\pi \sigma^{*}$ conical intersection lies close to the Franck-Condon structure, i.e., the equilibrium structure of $\mathrm{S}_{0}$ as shown in Fig. 2 (though the structure of this conical intersection is planar).

It is found from Tables 1 and 2 that this $\pi \pi^{*}{ }^{1} \mathrm{~L}_{\mathrm{a}}-\pi \sigma^{*}$ conical intersection lies higher than the $\pi \pi^{*}{ }^{1} \mathrm{~L}_{\mathrm{a}}$ state at the Franck-Condon geometry, by $0.19 \mathrm{eV}$ according to the present MRCI calculation and it is higher by $0.30 \mathrm{eV}$ than the potential minimum of the $\pi \pi^{*}{ }^{1} \mathrm{~L}_{\mathrm{a}}$ state. The transition from the ${ }^{1} \mathrm{~L}_{\mathrm{a}}$ state to the higher $\pi \sigma^{*}$ is presumably dominated by this conical intersection, although the ${ }^{1} \mathrm{~L}_{\mathrm{a}}$ state has a barrier of $0.25 \mathrm{eV}$ between the Franck-Condon region and the $\pi \pi^{*}$ ${ }^{1} L_{a}-\pi \sigma^{*}$ conical intersection (see Fig. 10(a)). This result is consistent with the experimental observation that an additional channel, besides the $n \pi^{*}$ pathway, is open at the excess energy of $\sim 0.2 \mathrm{eV}$ above the band origin of the $\pi \pi^{*}{ }^{1} \mathrm{~L}_{\mathrm{a}}$ state. ${ }^{3 e}$

Fig. $3 b$ shows the $g$-vector of the $\pi \pi^{*}{ }^{1} \mathrm{~L}_{\mathrm{a}}-\pi \sigma^{*}$ conical intersection. The main components of the $\boldsymbol{g}$-vector are in-plane stretching and distortion of the five and six-membered rings rather than the stretching of $\mathrm{N} 9-\mathrm{H} 14$. In our previous two-dimensional model for the $\pi \pi^{*}{ }^{1} \mathrm{~L}_{\mathrm{a}}-\pi \sigma^{*}$ conical intersection, ${ }^{18}$ the N9-H14 bond was taken as the reaction coordinate. The $\boldsymbol{g}$-vector indicates the direction of structure distortion toward the conical intersection. For instance, the $\boldsymbol{g}$-vector in Fig. 3b points to shortening of the N1-C6 bond length; the N1-C6 bond length $(1.29 \AA)$ at the $\pi \pi^{*}{ }^{1} \mathrm{~L}_{\mathrm{a}}-\pi \sigma^{*}$ conical intersection is shorter by $0.05 \AA$ than the length in $\mathrm{S}_{0}$ (See Fig. 2).

Fig. 3c shows the $\boldsymbol{h}$-vector of the $\pi \pi^{*}{ }^{1} L_{a}-\pi \sigma^{*}$ conical intersection. This Figure indicates that the $\boldsymbol{h}$-vector consists of ring puckering modes of the six-membered ring. We have decomposed the $\boldsymbol{h}$-vector to see the contributions of individual normal modes. The $\boldsymbol{h}$-vector can be expanded in terms of unit vectors of normal modes as $\boldsymbol{h}=\Sigma C_{\mathrm{i}} \boldsymbol{u}_{i}$. Here, $\boldsymbol{u}_{i}$ is the unit vector of $i$-th normal mode. The coefficient $C_{\mathrm{i}}$ is obtained as $C_{\mathrm{i}}=\boldsymbol{h} \cdot \boldsymbol{u}_{i}$. The analysis of the $\boldsymbol{h}$-vector of the $\pi \pi^{*}{ }^{1} \mathrm{~L}_{\mathrm{a}}-\pi \sigma^{*}$ conical intersection shows that $\boldsymbol{h} \propto-0.76 \boldsymbol{u}_{10}+0.42 \boldsymbol{u}_{20}+\cdots$. Normal modes $\nu_{10}$ and $\nu_{20}$ are out-of-plane bending modes and calculated frequencies in the ground state are 614 and $1117 \mathrm{~cm}^{-1}$, respectively. These normal modes mainly consist of out-of-plane vibrations of the C2-H13, N9-H14, C8-H15 and $\mathrm{C} 2-\mathrm{N} 3$ groups. This supports the choice of the coupling mode in our previous two-dimensional model, ${ }^{18}$ where the out-of-plane mode of the hydrogen atom (H14) was taken as the coupling mode.

3.3.2 The transfer stage. We now consider the transfer stage of the $\pi \sigma^{*}$ pathway which ranges from the $\pi \pi^{*}{ }^{1} \mathrm{~L}_{\mathrm{a}}-\pi \sigma^{*}$ conical intersection to the $\pi \sigma^{*}-\mathrm{S}_{0}$ conical intersection. The second conical intersection has a geometrical structure of $\mathrm{C}_{\mathrm{s}}$ symmetry as shown in Fig. 4a. It should be pointed out that $R_{\mathrm{N} 9 \mathrm{H}}=1.574 \AA$ is much longer than $R_{\mathrm{N} 9 \mathrm{H}}=0.993 \AA$ at the $\pi \pi^{* 1} \mathrm{~L}_{\mathrm{a}}-\pi \sigma^{*}$ conical intersection. This elongation is correlated with the observation of $\mathrm{H}$-atom fragments from adenine excited at $239.5 \mathrm{~nm}^{8 b}$ The present value of $R_{\mathrm{N} 9 \mathrm{H}}=1.574 \AA$ is slightly shorter than the CASSCF value $R_{\mathrm{N} 9 \mathrm{H}}=1.722 \AA$ for the two-dimensional model. ${ }^{18}$

The $g$-vector at the $\pi \sigma^{*}-\mathrm{S}_{0}$ conical intersection is shown in Fig. $4 b$, of which the components are almost localized on the N9-H14 bond stretching with a minor contribution from the $\mathrm{C} 8-\mathrm{N} 7$ bond. This is consistent with the elongation of the N9-H14 bond at the $\pi \sigma^{*}-\mathrm{S}_{0}$ conical intersection. For the stage involving the $\pi \sigma^{*}-\mathrm{S}_{0}$ conical intersection, the $\boldsymbol{g}$-vector obtained in the full dimensional model validates the choice of

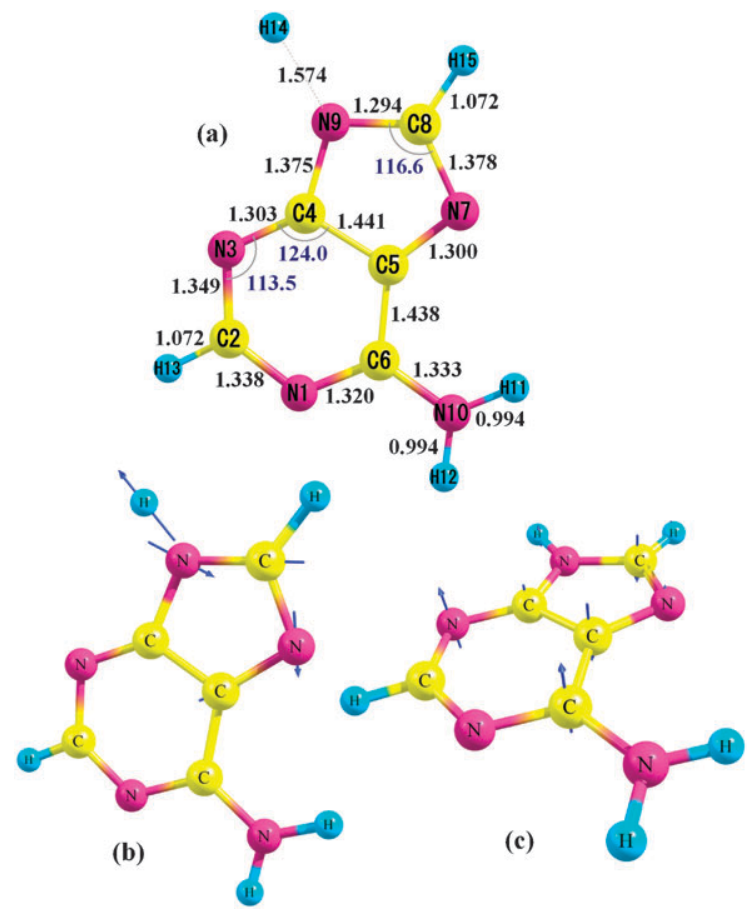

Fig. 4 (a) Cs structure at the $\pi \sigma^{*}-\mathrm{S}_{0}$ conical intersection, (b) the corresponding $\boldsymbol{g}$-vector and (c) $\boldsymbol{h}$-vector. 
the N9-H14 bond stretching as the reaction coordinate in the two-dimensional model. ${ }^{18}$

Fig. 4c shows the $\boldsymbol{h}$-vector at the $\pi \sigma^{*}-S_{0}$ conical intersection. The $\boldsymbol{h}$-vector can be expressed in terms of the unit vectors of normal modes as $\boldsymbol{h} \propto-0.64 \boldsymbol{u}_{13}-0.54 \boldsymbol{u}_{12}-$ $0.4 \boldsymbol{u}_{10} \cdots$. Here, modes $\nu_{12}$ and $\nu_{13}$ (the calculated frequencies in $S_{0}$ are 719 , and $767 \mathrm{~cm}^{-1}$ ) are also out-of-plane bending vibrations consisting of mainly atoms of the two rings.

The present calculation for the $\pi \sigma^{*}$ pathway indicates that a multidimensional picture is needed for accurate description of reaction and coupling coordinates, contrary to the picture of the single coupling mode in the simple two-dimensional model.

The MRCI energy is $4.13 \mathrm{eV}$ for the $\pi \sigma^{*}-\mathrm{S}_{0}$ conical intersection, while it is $5.32 \mathrm{eV}$ for the ${ }^{1} \mathrm{~L}_{\mathrm{a}}-\pi \sigma^{*}$ conical intersection. The potential energy curve along the LIIC from the $\pi \pi^{*}$ ${ }^{1} \mathrm{~L}_{\mathrm{a}}-\pi \sigma^{*}$ conical intersection to the $\pi \sigma^{*}-\mathrm{S}_{0}$ conical intersection is downhill. Therefore, the activation stage from the Franck-Condon geometry to the ${ }^{1} \mathrm{~L}_{\mathrm{a}}-\pi \sigma^{*}$ conical intersection is the rate-determining step in the $\pi \sigma^{*}$ pathway. Presumably, the main pathway from $\pi \sigma^{*}$ to $S_{0}$ is the one via this $\pi \sigma^{*}-S_{0}$ conical intersection: the trajectory passing by the planar geometry of the $\pi \pi^{*}{ }^{1} \mathrm{~L}_{\mathrm{a}}-\pi \sigma^{*}$ conical intersection can easily reach the $\pi \sigma^{*}-S_{0}$ conical intersection of planar geometry; the other conical intersections with $\mathrm{S}_{0}$ have nonplanar geometries. The $\pi \sigma^{*}$ pathway is isolated from the other relaxation pathways to $\mathrm{S}_{0}$.

\subsection{The $n \pi *$ pathway}

3.4.1 The activation stage. Let us consider the activation stage of the $n \pi^{*}$ pathway from the $\pi \pi^{*}{ }^{1} \mathrm{~L}_{\mathrm{a}}$ state to the $\pi \pi^{*}$ ${ }^{1} \mathrm{~L}_{\mathrm{a}}-n \pi^{*}$ conical intersection listed in Table 2. Fig. 5a shows the planar structure of this conical intersection which lies close to the Franck-Condon geometry. The energy of this conical

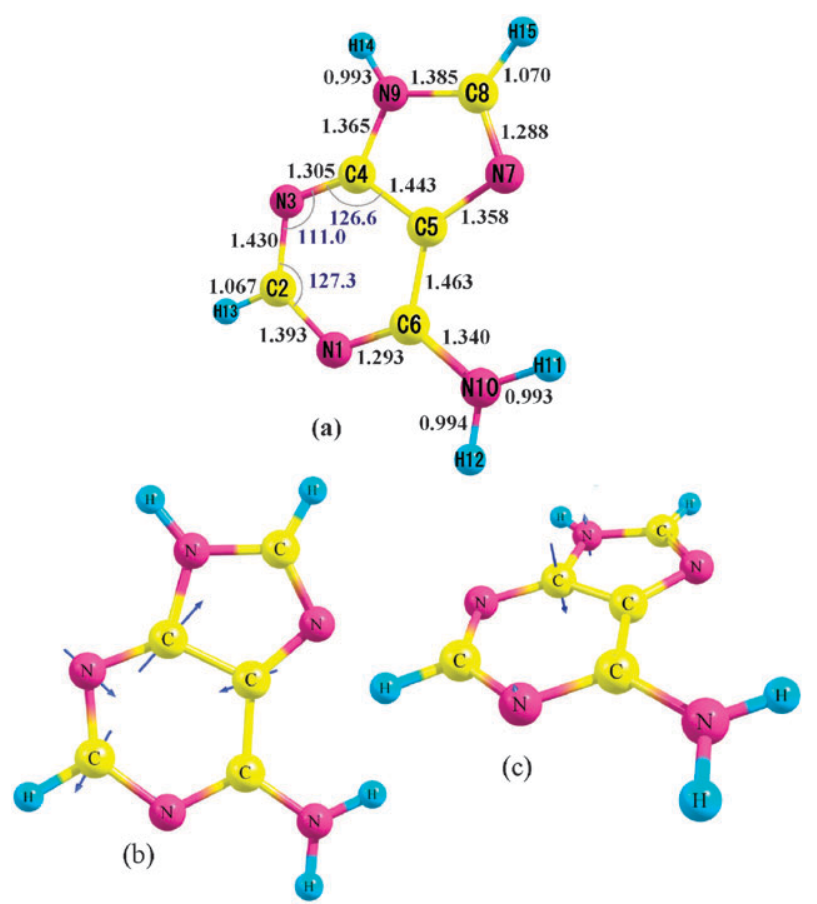

Fig. 5 (a) $\mathrm{C}_{\mathrm{s}}$ structure at the $\pi \pi^{* 1} \mathrm{~L}_{\mathrm{a}}-n \pi *$ conical intersection, (b) the corresponding $\boldsymbol{g}$-vector and (c) $\boldsymbol{h}$-vector. intersection is higher than the vertical excitation energy to the $\pi \pi^{* 1} \mathrm{~L}_{\mathrm{a}}$ state, only by $0.1 \mathrm{eV}$ (MRCI value).

According to the results of dynamics simulation by Barbatti and Lischka, ${ }^{17}$ the $\pi \pi^{*}{ }^{1} \mathrm{~L}_{\mathrm{a}}$ state relaxes to the lower electronic states, $n \pi^{*}$ and $\pi \pi^{*}{ }^{1} \mathrm{~L}_{\mathrm{b}}$, immediately after excitation (with a time constant of $22 \mathrm{fs}$ ). An ultrafast equilibration is achieved by the strong nonadiabatic couplings among the three states in the region where these states are located closely together. This is also supported by our MRCI result that the energy difference between the $\pi \pi^{*}{ }^{1} \mathrm{~L}_{\mathrm{a}}$ and $n \pi *$ states is smaller than $0.3 \mathrm{eV}$ in the vicinity of the Franck-Condon region and approaches zero at the $\pi \pi^{*}{ }^{1} \mathrm{~L}_{\mathrm{a}}-n \pi^{*}$ conical intersection found here. Ultrafast relaxation from $\pi \pi^{*}{ }^{1} \mathrm{~L}_{\mathrm{a}}$ to $n \pi^{*}$ would also occur around this conical intersection.

Fig. $5 \mathrm{~b}$ shows the $\boldsymbol{g}$-vector whose components are localized among $\mathrm{C} 2, \mathrm{C} 4, \mathrm{C} 5$ and $\mathrm{N} 3$ on the 6-membered ring. The $\boldsymbol{h}$-vector shown in Fig. 5c can be decomposed into normal modes as $\boldsymbol{h} \propto 0.44 \boldsymbol{u}_{5}-0.43 \boldsymbol{u}_{20}+0.41 \boldsymbol{u}_{10}-0.40 \boldsymbol{u}_{15}+0.34 \boldsymbol{u}_{13}-$ $0.31 \boldsymbol{u}_{12} \cdots$. Here, $\nu_{5}$ and $\nu_{15}$ (the calculated normal mode frequencies in the ground state are 326 and $891 \mathrm{~cm}^{-1}$ ) are out-of-plane bending modes. Note that the active coupling modes in this activation stage are distributed among several normal modes while the active coupling in the activation stage of the $\pi \sigma^{*}$ pathway is dominated by the out-of-plane normal mode $\nu_{10}$.

3.4.2 The transfer stage. The conical intersection between the $n \pi^{*}$ and $\mathrm{S}_{0}$ states is located at energy of $3.74 \mathrm{eV}$ (MRCI) as shown in Table 2. Fig. 6a shows that the geometrical structure of $9 \mathrm{H}$-adenine at the $n \pi^{*}-\mathrm{S}_{0}$ conical intersection belongs to $\mathrm{C}_{1}$ symmetry: the main deformation is of out-of-plane deformation of the 6-membered ring together with that of $\mathrm{NH}_{2}$ group, while the 5-membered ring keeps planar. The out-ofplane deformation is characterized by the following dihedral angles: $\delta(\mathrm{N} 3 \mathrm{C} 2 \mathrm{~N} 1 \mathrm{C} 6)=-48.9^{\circ}, \delta(\mathrm{C} 4 \mathrm{~N} 3 \mathrm{C} 2 \mathrm{~N} 1)=-7.80^{\circ}$,

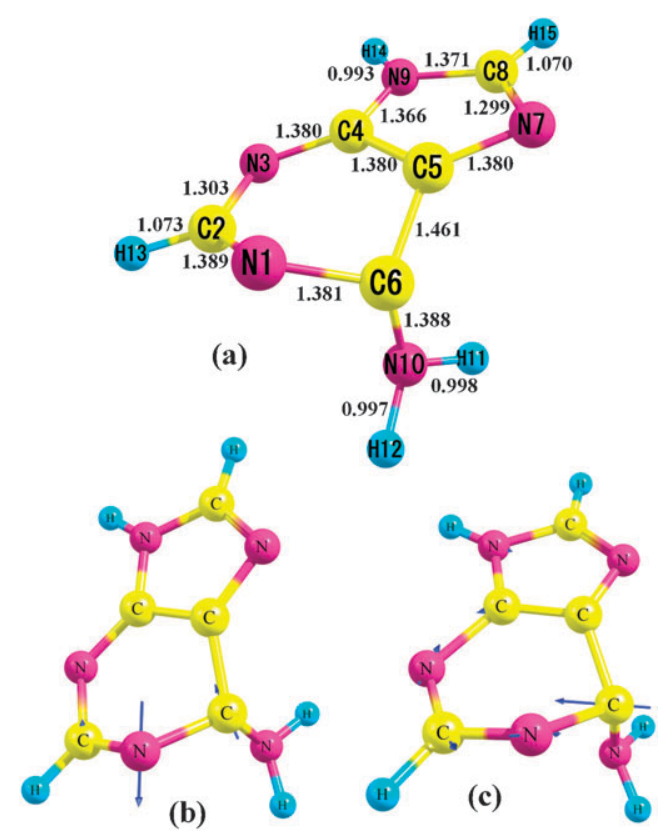

Fig. 6 (a) Nonplanar geometrical structure at the $n \pi^{*}-\mathrm{S}_{0}$ conical intersection, (b) the corresponding $\boldsymbol{g}$-vector and (c) $\boldsymbol{h}$-vector. 
$\delta(\mathrm{N} 9 \mathrm{C} 4 \mathrm{~N} 3 \mathrm{C} 2)=180.0^{\circ}, \delta(\mathrm{C} 8 \mathrm{~N} 9 \mathrm{C} 4 \mathrm{~N} 3)=-171.7^{\circ}$, $\delta(\mathrm{N} 10 \mathrm{C} 6 \mathrm{C} 5 \mathrm{~N} 7)=-63.8^{\circ}, \delta(\mathrm{N} 10 \mathrm{C} 6 \mathrm{~N} 1 \mathrm{C} 2)=-67.5^{\circ}$, $\delta(\mathrm{H} 13 \mathrm{C} 2 \mathrm{~N} 1 \mathrm{C} 6)=145.4^{\circ}$ and $\delta(\mathrm{H} 14 \mathrm{~N} 9 \mathrm{C} 4 \mathrm{~N} 3)=10.5^{\circ}$. Fig. $6 \mathrm{~b}$ and c show the $\boldsymbol{g}$-vector and $\boldsymbol{h}$-vector, respectively. The two vectors are not orthogonal to each other; many normal modes of in-plane and out-of-plane vibrations are mixed with each other. The potential energy curve along the LIIC in the transfer stage of the $n \pi^{*}$ pathway is also downhill $(5.23 \mathrm{eV}$ of the $\pi \pi^{* 1} \mathrm{~L}_{\mathrm{a}}-n \pi *$ conical intersection to $3.74 \mathrm{eV}$ of the $n \pi^{*}-\mathrm{S}_{0}$ conical intersection). Most of trajectories that hop from the $\pi \pi^{*}{ }^{1} \mathrm{~L}_{\mathrm{a}}$ to the diabatic $n \pi *$ state around the $\pi \pi^{*}{ }^{1} \mathrm{~L}_{\mathrm{a}}-n \pi^{*}$ conical intersection would reach the $n \pi^{*}-\mathrm{S}_{0}$ conical intersection.

\subsection{The direct pathway}

The direct pathway is defined as the diabatic relaxation route from the $\pi \pi^{*}{ }^{1} \mathrm{~L}_{\mathrm{a}}$ state via a conical intersection with $\mathrm{S}_{0}$. We found a $\pi \pi^{*}{ }^{1} \mathrm{~L}_{\mathrm{a}}-\mathrm{S}_{0}$ conical intersection in this pathway, of which the energy is $3.83 \mathrm{eV}$ at the CASSCF level of theory and $3.50 \mathrm{eV}$ at the MRCI (Table 2). The $\pi \pi^{*}{ }^{1} \mathrm{~L}_{\mathrm{a}}-\mathrm{S}_{0}$ conical intersection locates at the lowest energy among all the conical intersections in $9 \mathrm{H}$-adenine. The calculated geometrical structure at the $\pi \pi^{*}{ }^{1} \mathrm{~L}_{\mathrm{a}}-\mathrm{S}_{0}$ conical intersection is shown in Fig. 7a. The feature of the structure belonging to $C_{1}$ symmetry is in the out-of-plane deformation (puckered conformation) of the six-membered ring of $9 \mathrm{H}$-adenine, which is characterized by the following dihedral angles: $\delta(\mathrm{C} 4 \mathrm{~N} 3 \mathrm{C} 2 \mathrm{H} 13)=84.5^{\circ}$, $\delta(\mathrm{C} 6 \mathrm{~N} 1 \mathrm{C} 2 \mathrm{H} 13)=-77.0^{\circ}, \quad \delta(\mathrm{C} 5 \mathrm{C} 4 \mathrm{~N} 3 \mathrm{C} 2)=17.2^{\circ}$, $\delta(\mathrm{C} 5 \mathrm{C} 6 \mathrm{~N} 1 \mathrm{C} 2)=-22.3^{\circ}, \delta(\mathrm{N} 7 \mathrm{C} 5 \mathrm{C} 6 \mathrm{~N} 10)=7.3^{\circ}$.

Barbatti and Lischka ${ }^{17}$ have presented a systematic investigation of the seam of the conical intersections and classified geometrical structures of the six-membered ring of $9 \mathrm{H}$-adenine at the conical intersections by means of Cremer-Pople parameters: ${ }^{34}$ the geometrical structure of the lowest lying conical intersection is assigned as envelope ${ }^{2} \mathrm{E}$, i.e., an envelope puckering at the $\mathrm{C} 2$ atom. The structure in Fig. 7a corresponds to the envelope ${ }^{2} \mathrm{E}$ conformation found by them.

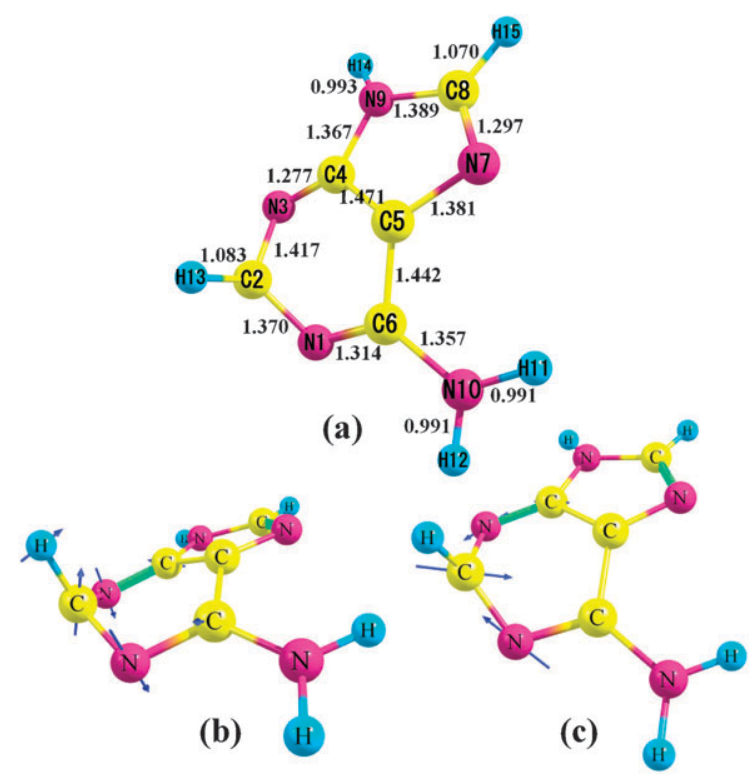

Fig. 7 (a) Nonplanar geometrical structure at the ${ }^{1} \mathrm{~L}_{\mathrm{a}}-\mathrm{S}_{0}$ conical intersection, (b) the corresponding $\boldsymbol{g}$-vector and (c) $\boldsymbol{h}$-vector.

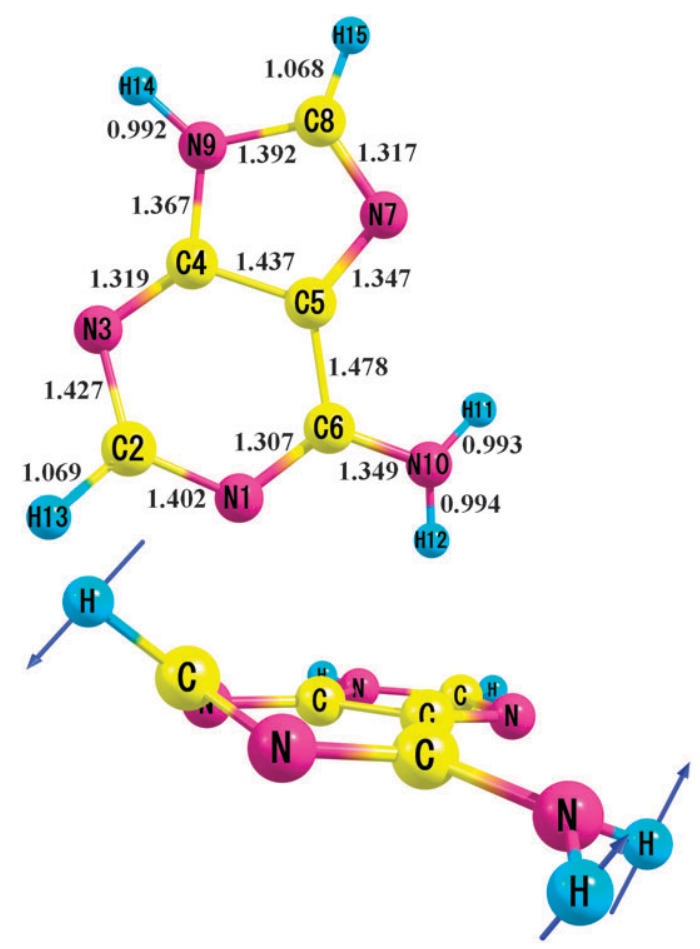

Fig. 8 Top and side view of the optimized transition state geometry in the ${ }^{1} \mathrm{~L}_{\mathrm{a}}$ state along the direct pathway. Bond lengths are in angstroms. The arrows indicate the mode for the imaginary frequency at the transition state.

The fact that the displacements of N1, C2 and N3 atoms make dominant contribution to the $\boldsymbol{g}$-vector in Fig. $7 \mathrm{~b}$ supports the envelope structure at the conical intersection.

As described in the Introduction, there have been hot discussions about whether or not the direct pathway is the dominant ultrafast relaxation process to $S_{0}$. We identified a transition state between the Franck-Condon geometry and the $\pi \pi^{*}{ }^{1} \mathrm{~L}_{\mathrm{a}}-\mathrm{S}_{0}$ conical intersection on the ${ }^{1} \mathrm{~L}_{\mathrm{a}}$ potential surface by using the quadratic steepest decent method. Here, the search was started at the geometry with the maximum energy along the LIIC connecting the Franck-Condon geometry and the $\pi \pi^{*}{ }^{1} \mathrm{~L}_{\mathrm{a}}-\mathrm{S}_{0}$ conical intersection. CASSCF calculations were performed: the energy of the obtained transition state is $8.23 \mathrm{eV}$ and it is $1.13 \mathrm{eV}$ higher than the vertical excitation energy.

The geometry of the transition state (shown in Fig. 8) is a midway configuration between the Franck-Condon geometry and the ${ }^{1} \mathrm{~L}_{\mathrm{a}}-\mathrm{S}_{0}$ conical intersection. The imaginary frequency mode obtained at the transition state, shown in the side view of Fig. 8, represents the motion between the two geometries. The geometry change is mainly in the dihedral angle H13C2N3C4; it is $180.3^{\circ}$ (almost planar) at the FranckCondon geometry, $140.3^{\circ}$ at the transition state geometry, and $83.4^{\circ}$ at the ${ }^{1} \mathrm{~L}_{\mathrm{a}}-\mathrm{S}_{0}$ conical intersection.

In order to more accurately estimate the barrier height of the transition state, we also performed single-point MRCI calculations for two linked LIICs; the LIIC connecting the Franck-Condon geometry and the transition state obtained at the CASSCF level of theory and the one connecting the transition state and the $\pi \pi^{*}{ }^{1} \mathrm{~L}_{\mathrm{a}}-\mathrm{S}_{0}$ conical intersection. The structure at the potential maximum obtained by this approach 
was identical to the transition state obtained by CASSCF. The MRCI energy of the transition state is $5.34 \mathrm{eV}$ and it is $0.21 \mathrm{eV}$ higher than the vertical excitation energy. The existence of a low barrier supports a previous result by Barbatti and Lischka ${ }^{17}$ that the barrier height for the diabatic pathway obtained by the LIIC method was as low as $0.04 \mathrm{eV}$ at the CASPT2 level of theory.

No other transition states were found between the Franck-Condon geometry and the $\pi \pi^{*}{ }^{1} \mathrm{~L}_{\mathrm{a}}-\mathrm{S}_{0}$ conical intersection on the diabatic $\pi \pi^{*}{ }^{1} \mathrm{~L}_{\mathrm{a}}$ surface. The trajectory of the direct pathway needs to reach the gate of this transition state and to go over it. Although the transition state may disappear at more accurate levels of theory, the intermediate "gate" in the direct pathway would be characterized by this transition state. This "gate" process competes with initial rapid equilibration among the three states ${ }^{1} \mathrm{~L}_{\mathrm{a}},{ }^{1} \mathrm{~L}_{\mathrm{b}}$, and $n \pi^{*}$. It is likely that a considerable number of trajectories circumvent the transition state and deviate from the direct ${ }^{1} \mathrm{~L}_{\mathrm{a}}$-diabatic pathway: the ${ }^{1} \mathrm{~L}_{\mathrm{a}}-n \pi^{*}$ conical intersection is located on the route to the transition state and the $n \pi^{*}$ and ${ }^{1} \mathrm{~L}_{\mathrm{a}}$ states are strongly coupled with each other in the region between the Franck-Condon geometry and the transition state geometry. Following this rapid scrambling, two routes through the $\pi \pi^{*}$ ${ }^{1} \mathrm{~L}_{\mathrm{a}}-\mathrm{S}_{0}$ and $n \pi^{*}-\mathrm{S}_{0}$ conical intersections are eventually open because the energy difference between the two conical intersections is as small as $0.24 \mathrm{eV}$ (MRCI). This scenario should be compared with the results of dynamics simulation by Barbatti and Lischka ${ }^{17}$ that most of the trajectories transfer to $\mathrm{S}_{0}$ via the lowest conical intersection, namely, the $\pi \pi^{*}$ ${ }^{1} \mathrm{~L}_{\mathrm{a}}-\mathrm{S}_{0}$ conical intersection, after rapid equilibration among the three states ${ }^{1} \mathrm{~L}_{\mathrm{a}},{ }^{1} \mathrm{~L}_{\mathrm{b}}$, and $n \pi^{*}$. Their MR-CIS calculation showed that the $\pi \pi^{*}{ }^{1} \mathrm{~L}_{\mathrm{a}}-\mathrm{S}_{0}$ conical intersection lies lower by $0.68 \mathrm{eV}$ than the $n \pi^{*}-\mathrm{S}_{0}$ conical intersection: the former conical intersection was more accessible than the latter.

\subsection{Geometric phase effects}

So far, we have discussed the conical intersections of $9 \mathrm{H}$-adenine from the energetic point of view. There is a way to unambiguously identify the conical intersections, that is, evaluation of the geometric (Berry) phase effects of adiabatic wavefunctions. This is based on the fact that the sign of the electronic wave function should be changed around a closed loop rotation near the vicinity of the conical intersection. We briefly explained the geometric phase effects in section 2.1. In this subsection, we confirm the existence of the conical intersections of $9 \mathrm{H}$-adenine from the view point of geometric phase effects.

The adiabatic wavefunctions $\Psi_{a}$ can be expanded in terms of various electronic configurations $\left\{\psi_{\alpha}(\theta)\right\}$ as a function of the position, denoted by angle $\theta$, on a closed loop around the conical intersection under consideration:

$$
\Psi_{a}=\sum_{\alpha} \chi_{a \alpha}(\theta) \psi_{\alpha}(\theta)
$$

where $\chi_{a \alpha}(\theta)$ are expansion coefficients. For a true conical intersection, the relation $\chi_{a \alpha}(\theta+2 \pi)=-\chi_{a \alpha}(\theta)$ should be satisfied. For $9 \mathrm{H}$-adenine, $\Psi_{a}$ are expressed in terms of 1764 configurations in the present CASSCF calculations. Here we show the coefficients of main four electronic configurations of interest, 22220000, 22211000, 21221000, and 22210100, where
0,1 , and 2 represent the occupation numbers of the eight active orbitals shown in Fig. 1. These are the main configurations of $\mathrm{S}_{0}, \pi \pi^{*}{ }^{1} \mathrm{~L}_{\mathrm{a}}, n \pi^{*}$ and $\pi \sigma^{*}$ states, respectively.

Fig. 9 shows the geometric phase effects of the conical intersections associated with the $n \pi *$ pathway and those with the direct pathway as examples. The higher and lower two adiabatic wavefunctions involved in the $\pi \pi^{*}{ }^{1} \mathrm{~L}_{\mathrm{a}}-n \pi^{*}$ conical intersection are denoted by $\Psi_{3}$ and $\Psi_{2}$, respectively. The coefficients of the four configurations in $\Psi_{2}$ and $\Psi_{3}$ are plotted in Fig. 9a against a closed loop rotation around the $\pi \pi^{*}$ ${ }^{1} \mathrm{~L}_{\mathrm{a}}-n \pi^{*}$ conical intersection. It is clearly demonstrated in the left panel of Fig. 9a that the coefficient of the 21221000 configuration ( $n \pi *$-character diabatic wavefunction) changes from 0.67 to -0.67 by the closed loop rotation. In the similar way, in the right panel of Fig. 9a, the coefficient of the 22211000 configuration $\left(\pi \pi^{*}{ }^{1} \mathrm{~L}_{\mathrm{a}}\right.$-character diabatic wavefunction) changes from -0.64 to 0.64 . The coefficients in the lowest two adiabatic wavefunctions $\left\{\Psi_{0}\right.$ and $\left.\Psi_{1}\right\}$ were not shown here since there are no geometric phase effects on them. Thus, the presence of the geometric phase effects supports that the conical intersection originates from a true potential crossing between the $\pi \pi^{*}{ }^{1} \mathrm{~L}_{\mathrm{a}}$ and $n \pi *$ adiabatic states.

For the $n \pi^{*}-\mathrm{S}_{0}$ conical intersection, we note that the original $\boldsymbol{g}$ - and $\boldsymbol{h}$-vectors are not orthogonal to each other because of its nonplanar geometrical structure (See Fig. 6a). Therefore, they cannot be used as orthogonal bases for rotation. To overcome this problem, the orthogonalization method described in section 2.1 was adopted. ${ }^{19 b, c}$ The geometric phase effects of the $n \pi^{*}-\mathrm{S}_{0}$ conical intersection are confirmed by a closed loop rotation in terms of the newly orthogonalized vectors, as shown in Fig. 9b. In the left panel of Fig. 9b, the coefficient of the 22220000 configuration ( $\mathrm{S}_{0}$-character diabatic wavefunction) changes from 0.93 to -0.93 by the closed loop rotation. In the right panel of Fig. $9 b$, the coefficient of the 21221000 configuration $\left(n \pi^{*}\right.$-character diabatic wavefunction) changes from -0.63 to 0.63 . The results clearly indicates that this conical intersection originates from a potential crossing between the $n \pi *$ and $\mathrm{S}_{0}$ states.

The same treatment has been done to confirm the conical intersection for the direct pathway ${ }^{1} \mathrm{~L}_{\mathrm{a}} \rightarrow \mathrm{S}_{0}$, where again the $\boldsymbol{g}$ and $\boldsymbol{h}$ vectors are not orthogonal to each other and need to be orthogonalized first. In the left panel of Fig. 9c, the coefficient of the 22220000 configuration ( $\mathrm{S}_{0}$-character diabatic wavefunction) changes from -0.69 to 0.69 by a closed loop rotation in terms of the newly orthogonalized vectors. In the right panel of Fig. 9c, the coefficient of 22211000 configuration $\left({ }^{1} \mathrm{~L}_{\mathrm{a}}\right.$-character diabatic wavefunction) changes from 0.65 to -0.65 . The results clearly indicate that this conical intersection originates from a potential crossing between the ${ }^{1} L_{a}$ and $S_{0}$ states. We have also confirmed the geometric phase effects on the conical intersections associated with the $\pi \sigma^{*}$ state.

\subsection{Control of radiationless transition pathway}

It is possible to discuss which pathway is more favorable between the $\pi \sigma^{*}$ and $n \pi^{*}$ pathways by comparing their activation energy $\Delta E$. Ullrich, Schultz, Zgiersky and Stolow, ${ }^{3 e}$ and Samoylova et al. ${ }^{10 d}$ have carried out time-resolved experiments of $9 \mathrm{H}$-adenine in a molecular beam and measured the 

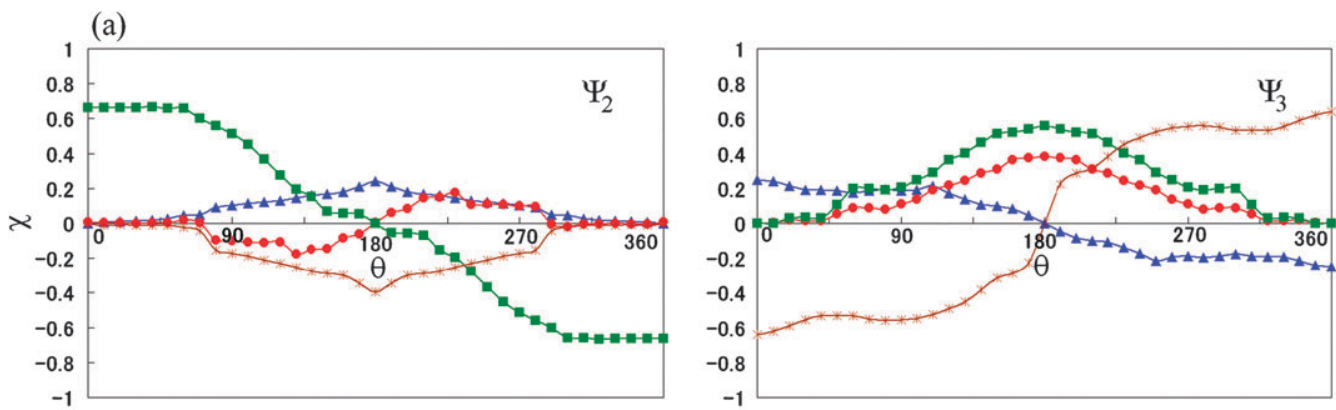

(b)
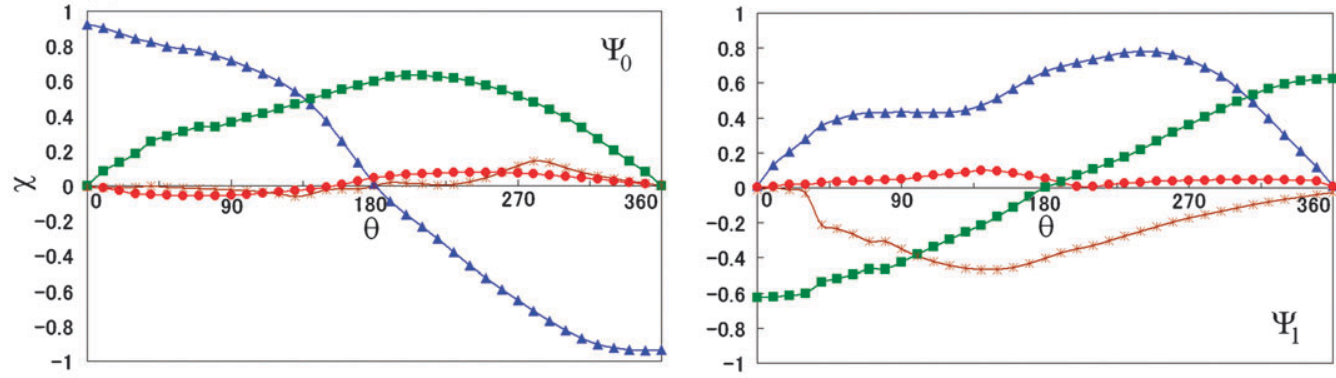

(c)
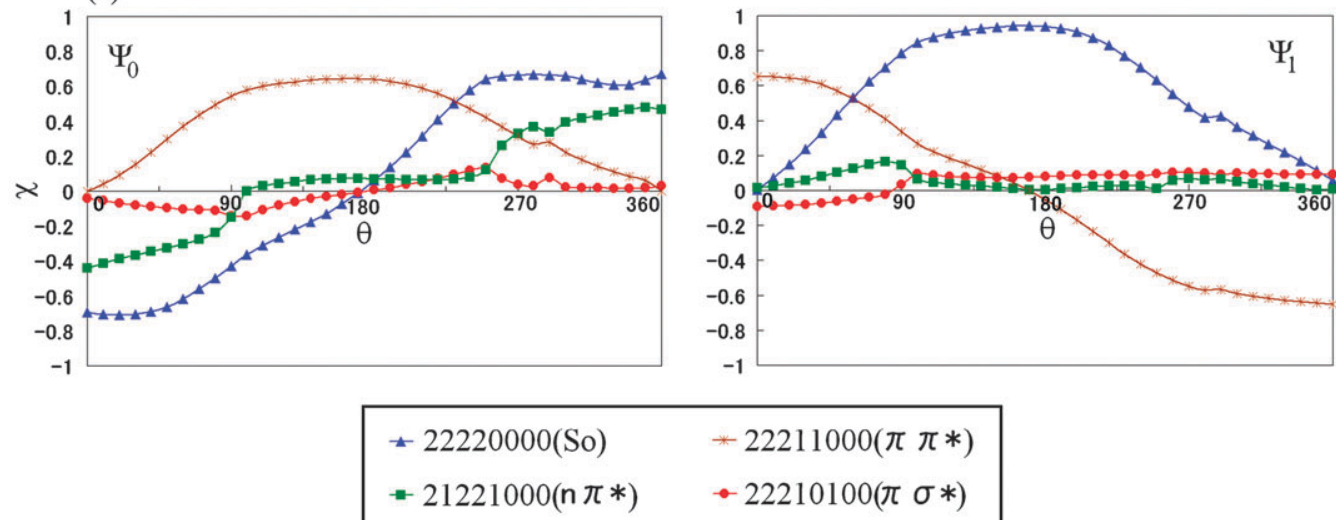

$* 22211000(\pi \pi *)$

$-22210100(\pi \sigma *)$

Fig. 9 Geometric phase effects on (a) the $\pi \pi^{*}{ }^{1} \mathrm{~L}_{\mathrm{a}}-n \pi^{*}$ conical intersection, (b) the $n \pi *-\mathrm{S}_{0}$ conical intersection, and (c) the $\pi \pi^{*}{ }^{1} \mathrm{~L}_{\mathrm{a}}-\mathrm{S}_{0}$. The adiabatic wavefunctions $\left\{\Psi_{a}\right\}$ are expanded in terms of four electronic configurations $\left\{\psi_{\alpha}\right\}$ denoted by $22220000,22211000,21221000$, and 22210100 , which are the main electron configurations of the $\mathrm{S}_{0}, \pi \pi^{* 1} \mathrm{~L}_{\mathrm{a}}, n \pi^{*}$ and $\pi \sigma^{*}$ states, respectively. The coefficients $\left\{\chi_{a \alpha}\right\}$ are plotted as a function of the closed loop angle $\theta$ around the conical intersection.

excitation energy dependence of the branching ratio between the $\pi \sigma^{*}$ and $n \pi^{*}$ pathways. They suggested that the $\pi \sigma^{*}$ pathway contributes to ultrafast relaxation from $\pi \pi^{*}{ }^{1} \mathrm{~L}_{\mathrm{a}}$ at higher excess energies from the absorption band origin $(>0.2 \mathrm{eV})$, which agrees with our MRCI result that the $\pi \pi^{*}$ ${ }^{1} L_{a}-\pi \sigma^{*}$ conical intersection lies higher by $0.19 \mathrm{eV}$ than the $\pi \pi^{* 1} L_{\mathrm{a}}$ state at the Franck-Condon geometry. However, our present MRCI level of calculation indicates that the activation energies of the three pathways are close to each other; the conical intersections in the activation stages of the $\pi \sigma^{*}$ and $n \pi^{*}$ pathways are only slightly higher (by $0.1-0.2 \mathrm{eV}$ ) than the $\pi \pi^{* 1} \mathrm{~L}_{\mathrm{a}}$ state at the Franck-Condon geometry. Therefore, it is difficult to precisely determine from the obtained activation energies which of the $\pi \sigma^{*}$ and $n \pi^{*}$ pathways is more dominant. Nevertheless, we can consider a scenario for controlling favorable radiationless transition pathways in the activation stages on the basis of the analysis of $\boldsymbol{h}$-vectors that different pathways have different coupling modes.
In section 3.3 and 3.4, we have expanded the $\boldsymbol{h}$-vector for each conical intersection in terms of normal modes. The $\boldsymbol{h}$-vector in the activation stage of the $\pi \sigma^{*}$ pathway shown in Fig. $3 \mathrm{c}$ are significantly different from those in the activation stage of the $n \pi^{*}$ pathway shown in Fig. $5 \mathrm{c}$ : the active coupling for the $\pi \sigma^{*}$ pathway is dominated by the out-of-plane normal mode $\nu_{10}$, while the active coupling for the $n \pi *$ pathway is distributed among many normal modes. Thus, the coupling modes in the two pathways are only weakly mixed with each other. This suggests that the branching ratio of the two pathways can be controlled by selective excitation of single vibronic levels involving active coupling modes such as the mode $\nu_{10}$. A realistic way to achieve this is to use IR-UV double resonance techniques. More elaborate scenarios can be proposed on the basis of quantum control theory, a powerful tool for designing optimal pulses to control the branching ratios of dynamical pathways. Here, quantum control should be carried out on the whole radiationless processes involving both 
the activation and transfer stages as in a previous paper on the control of the $\pi \sigma^{*}$ pathway in the two-dimensional model. ${ }^{35}$

\section{Summary and conclusions}

CASSCF and MRCI levels of calculations with the 6-31++ $\mathrm{G}^{* *}$ basis set were carried out to determine effective pathways of ultrafast radiationless transitions from the optically allowed $\pi \pi^{*}{ }^{1} \mathrm{~L}_{\mathrm{a}}$ state of $9 \mathrm{H}$-adenine. The $n \pi^{*}, \pi \sigma^{*}$ and $\pi \pi^{*}$ states were considered as states involved in the radiationless process. We searched conical intersections in the full dimensional space by using the CASSCF level of calculation installed in MOLPRO. The related energies were refined by a single-point $\mathrm{MRCI}^{24}$ calculation. The MRCI transition energies to excited states are in good agreement with the experimental values, as shown in Table 1. By identifying the structures and energies of conical intersections involved in ultrafast radiationless transitions from ${ }^{1} \mathrm{~L}_{\mathrm{a}}$ to $\mathrm{S}_{0}$, we examined the mechanisms of three competing pathways; two indirect pathways via the $\pi \sigma^{*}$ and $n \pi^{*}$ states, ${ }^{1} \mathrm{~L}_{\mathrm{a}} \rightarrow \pi \sigma^{*} \rightarrow \mathrm{S}_{0}$ ( $\pi \sigma^{*}$ pathway) and ${ }^{1} \mathrm{~L}_{\mathrm{a}} \rightarrow n \pi^{*} \rightarrow \mathrm{S}_{0}$ ( $n \pi^{*}$ pathway), and the direct pathway ${ }^{1} \mathrm{~L}_{\mathrm{a}} \rightarrow \mathrm{S}_{0}$. Schematic illustrations for the three pathways are presented in Fig. 10. Geometric phase effects of the adiabatic electronic wavefunctions have been evaluated to confirm that the potential crossing is a true conical intersection. We have confirmed that the $n \pi *$ and $\pi \sigma^{*}$ pathways are independent of each other because the non-adiabatic coupling between the $\pi \sigma^{*}$ and $n \pi^{*}$ states is negligibly small.

The radiationless process of ${ }^{1} \mathrm{~L}_{\mathrm{a}} \rightarrow \pi \sigma^{*}$ in the $\pi \sigma^{*}$ pathway is dominated by the $\pi \pi^{*}{ }^{1} \mathrm{~L}_{\mathrm{a}}-\pi \sigma^{*}$ conical intersection which lies higher than the $\pi \pi^{*}{ }^{1} \mathrm{~L}_{\mathrm{a}}$ state at the Franck-Condon geometry, by $0.19 \mathrm{eV}$, according to the present MRCI calculation. The existence of this conical intersection is consistent with the experimental observation that a new channel is open at the excess energy of $\sim 0.2 \mathrm{eV}$ above the band origin of the $\pi \pi^{*}{ }^{1} \mathrm{~L}_{\mathrm{a}}$ state. ${ }^{3 e}$ The potential energy curve along the LIIC from the $\pi \pi^{*}{ }^{1} \mathrm{~L}_{\mathrm{a}}-\pi \sigma^{*}$ conical intersection to the $\pi \sigma^{*}-\mathrm{S}_{0}$ conical intersection (transfer stage) is downhill. It should be also noted that the ${ }^{1} \mathrm{~L}_{\mathrm{a}}-\pi \sigma^{*}$ and $\pi \sigma^{*}-\mathrm{S}_{0}$ conical intersections are connected to each other on the plane. We therefore conclude that relaxation from the transfer stage to $S_{0}$ occurs mainly through the $\pi \sigma^{*}-\mathrm{S}_{0}$ conical intersection.

We also located the conical intersection involved in the $n \pi *$ pathway. The $\pi \pi^{*}{ }^{1} \mathrm{~L}_{\mathrm{a}}-n \pi^{*}$ conical intersection is of planar conformation and located close to the Franck-Condon geometry. This conical intersection lies higher only by $0.1 \mathrm{eV}$ (MRCI) than the $\pi \pi^{*}{ }^{1} \mathrm{~L}_{\mathrm{a}}$ state at the Franck-Condon geometry and would contributes to rapid radiationless transitions from the initially prepared $\pi \pi^{*}{ }^{1} \mathrm{~L}_{\mathrm{a}}$ state (which correspond to the fast decay component in time-resolved spectra of $9 \mathrm{H}$-adenine). The subsequent process is the relaxation to $\mathrm{S}_{0}$ via the $n \pi *-\mathrm{S}_{0}$ conical intersection of $\mathrm{C}_{1}$ symmetry which lies lower by $1.49 \mathrm{eV}$ than the $\pi \pi^{*}{ }^{1} \mathrm{~L}_{\mathrm{a}}-n \pi *$ conical intersection.

The $\pi \pi^{*}{ }^{1} \mathrm{~L}_{\mathrm{a}}-\mathrm{S}_{0}$ conical intersection in the direct pathway has the lowest energy among the conical intersections found in this study. We identified the transition state between the Franck-Condon geometry and the $\pi \pi^{*}{ }^{1} \mathrm{~L}_{\mathrm{a}}-\mathrm{S}_{0}$ conical intersection on the ${ }^{1} \mathrm{~L}_{\mathrm{a}}$ potential surface. The MRCI energy of the transition state is higher by $0.21 \mathrm{eV}$ than the vertical excitation energy. No other transition states were found between the Franck-Condon geometry and the $\pi \pi^{*}{ }^{1} \mathrm{~L}_{\mathrm{a}}-\mathrm{S}_{0}$ conical intersection. The ${ }^{1} \mathrm{~L}_{\mathrm{a}}-n \pi *$ conical intersection is located on the route to the transition state. Moreover, the $n \pi *$ and ${ }^{1} \mathrm{~L}_{\mathrm{a}}$ states can be strongly coupled with each other because of the small energy gap in the region between the Franck-Condon geometry and the transition state geometry. It is therefore likely that a considerable number of trajectories deviate from the direct ${ }^{1} \mathrm{~L}_{\mathrm{a}}$-diabatic pathway. Following this rapid scrambling, two routes through the $\pi \pi^{*}{ }^{1} \mathrm{~L}_{\mathrm{a}}-\mathrm{S}_{0}$ and $n \pi *-\mathrm{S}_{0}$ conical intersections are eventually open: the energy difference between the two conical intersections is as small as $0.24 \mathrm{eV}$ (MRCI). Presumably, these two routes correspond to the slow decay component because the $\pi \pi^{*}{ }^{1} \mathrm{~L}_{\mathrm{a}}-\mathrm{S}_{0}$ and $n \pi *-\mathrm{S}_{0}$ conical intersections are subject to large out-of-plane distortion while the structures in the activation stages are almost planar.

The $\boldsymbol{g}$ - and $\boldsymbol{h}$-vectors defined at the conical intersections are projected onto normal modes to check the validity of the two-dimensional model for the $\pi \sigma^{*}$ pathway, where the N9-H14 bond was taken as the $\boldsymbol{g}$-vector (tuning mode) and the out-of-plane mode of H14 was taken as the $\boldsymbol{h}$-vector (coupling mode). ${ }^{18}$ Our full dimensional search has shown for the $\pi \pi^{*}{ }^{1} \mathrm{~L}_{\mathrm{a}}-\pi \sigma^{*}$ conical intersection that the main components of the $\boldsymbol{g}$-vector are in-plane stretching and distortion of the five and six-membered rings rather than the stretching of N9-H14 while the $\boldsymbol{h}$-vector mainly consist of out-of-plane modes including H14. The $\boldsymbol{g}$ - and $\boldsymbol{h}$-vectors for the $\pi \sigma^{*}-\mathrm{S}_{0}$ conical intersection, which are orthogonal to each other, consist of many normal modes of in-plane and out-ofplane vibrations. The simple two-dimensional model cannot give an essential description of the $\pi \sigma^{*}$ pathway.

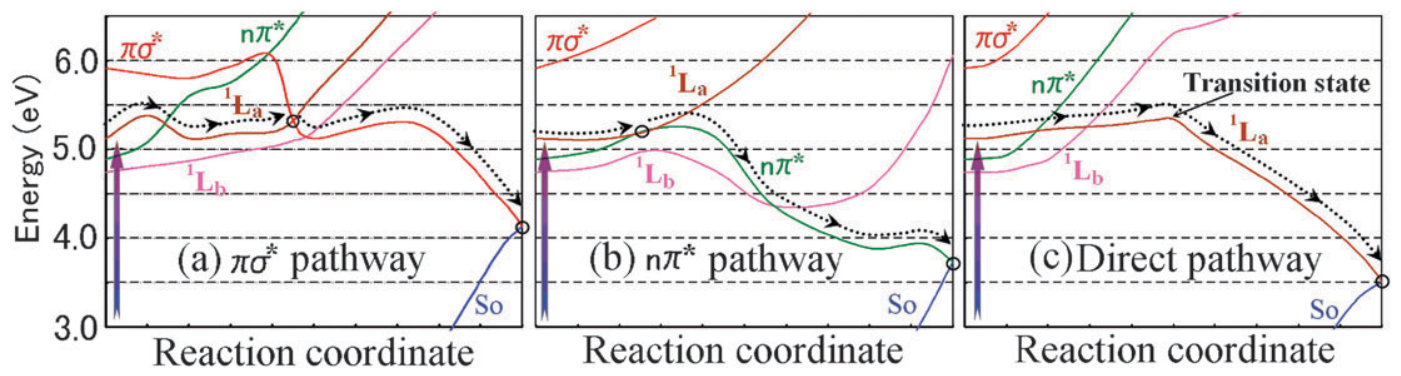

Fig. 10 Potential energy surfaces at the MRCI level of calculation for the three pathways: (a) the $\pi \sigma^{*}$ pathway; (b) the $n \pi^{*}$ pathway and (c) the direct pathway. The circles indicate the positions of the conical intersections. The vertical arrow in each panel represents the optical excitation from the potential minimum of the $S_{0}$ state to the ${ }^{1} L_{a} \pi \pi^{*}$. The origin of the energy is the potential minimum of $S_{0}$. 
The main coupling modes are extracted from the $\boldsymbol{h}$-vector for each conical intersection. The active coupling for the activation stage of the $\pi \sigma^{*}$ pathway is dominated by the out-of-plane normal mode $\nu_{10}$, while the active coupling for the $n \pi^{*}$ pathway is distributed among many normal modes. We proposed a scenario to enhance a specific pathway; single vibronic levels involving different active coupling modes can be selectively prepared by IR-UV double resonance.

\section{Acknowledgements}

WMIH thanks a Japanese Government (Monbukagakusho) DSc Scholarship and Tohoku Kaihatsu memorial foundation for his support. We thank Professors W. Domcke and S. H. Lin for their critical comments. This research was partially supported by the Ministry of Education, Science, Sports and Culture, Grant-in-Aid for Scientific Research on Priority Areas, "Molecular Theory for Real Systems" (No. 461).

\section{References}

1 (a) M. Daniels, in Photochemistry and Photobiology of Nucleic Acids, ed. S. Y. Wang, Academic Press, New York, 1976, vol. 1, p. 23; (b) P. R. Callis, Annu. Rev. Phys. Chem., 1983, 34, 329; (c) J. Cadet, W. Roth and I. Fischer, in Bioorganic Photochemistry, ed. H. Morrison, John Wiley \& Sons, New York, 1990, vol. 1, p. 1; (d) M. Daniels and W. Hauswirt, Science, 1971, 171, 675.

2 (a) C. E. Crespo-Hernández, B. Cohen, P. M. Hare and B. Kohler, Chem. Rev., 2004, 104, 1977; (b) B. Cohen, P. M. Hare and B. Kohler, J. Am. Chem. Soc., 2003, 125, 13594; (c) H. Saigusa, J. Photochem. Photobiol., 2007, 7, 197; references therein.

3 (a) D. C. Lührs, J. Viallon and I. Fischer, Phys. Chem. Chem. Phys., 2001, 3, 1827; (b) E. Nir, K. Kleinermanns, L. Grace and M. S. De Vries, J. Phys. Chem. A, 2001, 105, 5106; (c) C. Plützer and K. Kleinermanns, Phys. Chem. Chem. Phys., 2002, 4, 4877; (d) H. Kang, B. Jung and S. K. Kim, J. Chem. Phys., 2003, 118, 6717; (e) S. Ullrich, T. Schultz, M. Z. Zgierski and A. Stolow, J. Am. Chem. Soc., 2004, 126, 2262; (f) S. Ullrich, T. Schultz, M. Z. Zgierski and A. Stolow, Phys. Chem. Chem. Phys., 2004, 6, 2796; $(g)$ H. Satzger, D. Townsend, M. Zgierski, S. Patchkovskii, S. Ullrich and A. Stolow, Proc. Natl. Acad. Sci. U. S. A., 2006, 103, 10196.

4 G. Herzberg and H. C. Longuet-Higgins, Discuss. Faraday Soc., 1963, 35, 77-82.

5 W. Domcke, in Conical Intersections: Electronic Structure, Dynamic and Spectroscopy, ed. W. Domcke, D. Yarkony and H. Köppel, World Scientific, Singapore, 2004, vol. 15, ch. 9, p. 396.

6 (a) H. Köuppel, W. Domcke and L. S. Cederbaum, Adv. Chem. Phys., 1984, 57, 59; (b) W. Domcke, C. Woywod and M. Stengle, Chem. Phys. Lett., 1994, 226, 257; (c) C. Woywod, W. Domcke, A. L. Sobolewski and H. J. Werner, J. Chem. Phys., 1994, 100, 1400; (d) W. Domcke and G. Stock, in Adv. Chem. Phys., ed. I. Prigogine and S. A. Rice, J. Wiley, New York, 1997, vol. 100, p. 1; (e) Z. Lan, W. Domcke, V. Vallet, A. L. Sobolewski and S. Mahapatra, J. Chem. Phys., 2005, 122, 224315.

7 (a) A. L. Sobolewski and W. Domcke, Eur. Phys. J. D, 2002, 20, 369; (b) A. L. Sobolewski, W. Domcke, C. Dedonder-Lardeux and C. Jouvet, Phys. Chem. Chem. Phys., 2002, 4, 1093.

8 (a) I. Hünig, C. Plützer, K. A. Seefeld, D. Löwenich, M. Nispel and K. Kleinermanns, ChemPhysChem, 2004, 5, 1427; (b) M. Zierhut, W. Roth and I. Fischer, Phys. Chem. Chem. Phys., 2004, 6, 5178.

9 K. L. Wells, G. M. Roberts and V. G. Stavros, Chem. Phys. Lett., 2007, 446, 20.

10 (a) H. Satzger, D. Townsend and A. Stolow, Chem. Phys. Lett., 2006, 430, 144; (b) C. Canuel, M. Mons, F. Piuzzi, B. Tardivel, I. Dimicoli and M. Elhanine, J. Chem. Phys., 2005, 122, 074316; (c) H.-H. Ritze, H. Lippert, E. Samoylova, V. R. Smith, I. V. Hertel, W. Radloff and T. Shlutz, J. Chem. Phys., 2005, 122, 224320; (d) E. Samoylova, H. Lippert, S. Ullrich, I. V. Hertel, W. Radloff and T. Schultz, J. Am. Chem. Soc., 2005, 127, 1782.
11 (a) S. Perun, A. L. Sobolewski and W. Domcke, Chem. Phys., 2005, 313, 107; (b) S. Perun, A. L. Sobolewski and W. Domcke, J. Am. Chem. Soc., 2005, 127, 6257; (c) S. Perun, A. L. Sobolewski and W. Domcke, Mol. Phys., 2006, 104, 1113.

12 H. Chen and S. Li, J. Phys. Chem. A, 2005, 109, 8443.

13 C. H. Chin, A. M. Mebel, G. S. Kim, K. Y. Baek, M. Hayashi, K. K. Liang and S. H. Lin, Chem. Phys. Lett., 2007, 445, 361.

14 L. Blancafort, J. Am. Chem. Soc., 2006, 128, 210.

15 (a) L. Serrano-Andrés, M. Merchán and A. C. Borin, Chem.-Eur. J., 2006, 12, 6559; (b) L. Serrano-Andrés, M. Merchán and A. C. Borin, Proc. Natl. Acad. Sci. U. S. A., 2006, 103, 8691.

16 E. Fabiano and W. Thiel, J. Phys. Chem. A, 2008, 112, 6859.

17 M. Barbatti and H. Lischka, J. Am. Chem. Soc., 2008, 130, 6831.

18 W. C. Chung, Z. Lan, Y. Ohtsuki, N. Shimakura, W. Domcke and Y. Fujimura, Phys. Chem. Chem. Phys., 2007, 9, 2075.

19 (a) D. R. Yarkony, Acc. Chem. Res., 1998, 31, 511; (b) D. R. Yarkony, J. Chem. Phys., 2000, 112, 2111; (c) D. R. Yarkony, J. Phys. Chem. A, 2001, 105, 6277.

20 S. Matsika, J. Phys. Chem. A, 2005, 109, 7538.

21 R. D. Amos, A. Bernhardsson, A. Berning, P. Celani, D. L. Cooper, M. J. O. Deegan, A. J. Dobbyn, F. Eckert, C. Hampel, G. Hetzer, P. J. Knowles, T. Korona, R. Lindh, A. W. Lloyd, S. J. McNicholas, F. R. Manby, W. Meyer, M. E. Mura, A. Nicklass, P. Palmieri, R. Pitzer, G. Rauhut, M. Schütz, U. Schumann, H. Stoll, A. J. Stone, R. Tarroni, T. Thorsteinsson and H.-J. Werner, MOLPRO, a package of ab initio programs designed by H.-J. Werner and P. J. Knowles, Version 2002.1, 2002.

22 (a) H. C. Longuet-Higgins, Adv. Spectrosc., 1961, 2, 429; (b) M. V. Berry, Proc. R. Soc. London, Ser. A, 1984, 392, 45.

23 (a) D. Hegarty and M. A. Robb, Mol. Phys., 1979, 38, 1795; (b) R. H. E. Eade and M. A. Robb, Chem. Phys. Lett., 1981, 83, 362; (c) H. B. Schlegel and M. A. Robb, Chem. Phys. Lett., 1982, 93, 43; (d) F. Bernardi, A. Bottini, J. J. W. McDougall, M. A. Robb and H. B. Schlegel, Faraday Symp. Chem. Soc., 1984, 19, 137; (e) N. Yamamoto, T. Vreven, M. A. Robb, M. J. Frisch and H. B. Schlegel, Chem. Phys. Lett., 1996, 250, 373; $(f)$ M. J. Frisch, I. N. Ragazos, M. A. Robb and H. B. Schlegel, Chem. Phys. Lett., 1992, 189, 524.

24 (a) H. J. Werner and P. J. Knowles, J. Chem. Phys., 1988, 89, 5803; (b) P. J. Knowles and H.-J. Werner, Chem. Phys. Lett., 1988, 145, 514.

25 G. Schaftenaar and J. H. Noordik, "Molden: a pre- and postprocessing program for molecular and electronic structures", J. Comput.-Aided Mol. Des., 2000, 14, 123.

26 S. Perun, A. L. Sobolewski and W. Domcke, J. Phys. Chem. A, 2006, 110, 13238.

27 W. Zierkiewicz, L. Komorowski, D. Michalska, J. Cerny and P. Hobza, J. Phys. Chem. B, 2008, 112, 16734.

28 (a) T. J. Kistenmacher and T. Shigematsu, Acta Crystallogr., Sect. B: Struct. Crystallogr. Cryst. Chem., 1974, 30, 1528; (b) R. K. McMullan, P. Benci and B. M. Craven, Acta Crystallogr., Sect. B: Struct. Crystallogr. Cryst. Chem., 1980, 36, 1424.

29 (a) A. Broo and A. Holmen, Chem. Phys., 1996, 211, 147; (b) J. M. Jean and K. B. Hall, J. Phys. Chem. A, 2000, 104, 1930; (c) J. Sponer, J. Florian, P. Hobza and J. Leszczynski, J. Biomol. Struct., 1996, 13, 827; (d) A. K. Chandra, M. T. Nguyen, T. Uchimaru and T. Zeegers-Hyskens, J. Phys. Chem. A, 1999, 103, 8853.

30 M. Eisenstein, Acta Crystallogr., Sect. B: Struct. Sci., 1988, 44, 412.

31 H. De Voe and I. Tinoco, Jr., J. Mol. Biol., 1962, 4, 500.

32 (a) L. B. Clark, G. G. Peschel and I. Tinoco, Jr., J. Phys. Chem., 1965, 69, 3615; (b) C. A. Sprecher and W. C. Johnson, Jr., Biopolymers, 1977, 16, 2243; (c) L. Li and D. M. Lubman, Anal. Chem., 1987, 59, 2538.

33 (a) S. Alavi, J. Phys. Chem. A, 2005, 109, 9536; (b) H. Berthod and A. Pullman, J. Chim. Phys., 1965, 62, 942; (c) H. Berthod and A. Pullman, C. R. Hebd. Seances Acad. Sci., 1963, 257, 2738; (d) M. P. Fülscher, L. Serrano-Andrés and B. O. Roos, J. Am. Chem. Soc., 1997, 119, 6168.

34 (a) D. Cremer and J. A. Pople, J. Am. Chem. Soc., 1975, 97, 1354; (b) J. C. A. Boeyens, J. Cryst. Mol. Struct., 1978, 8, 317.

35 (a) M. Abe, Y. Ohtsuki, Y. Fujimura and W. Domcke, J. Chem. Phys., 2005, 123, 144508; (b) M. Abe, Y. Ohtsuki, Y. Fujimura, Z. Lan and W. Domcke, J. Chem. Phys., 2006, 124, 224316. 